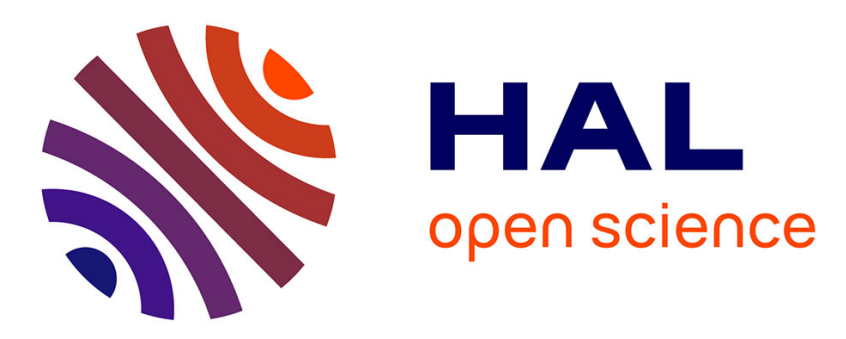

\title{
Active Tactile Transfer Learning for Object Discrimination in an Unstructured Environment using Multimodal Robotic Skin
}

\author{
Mohsen Kaboli, Di Feng, Gordon Cheng
}

\section{- To cite this version:}

Mohsen Kaboli, Di Feng, Gordon Cheng. Active Tactile Transfer Learning for Object Discrimination in an Unstructured Environment using Multimodal Robotic Skin. International Journal of Humanoid Robotics, 2017. hal-01609881

\section{HAL Id: hal-01609881 \\ https://hal.science/hal-01609881}

Submitted on 4 Oct 2017

HAL is a multi-disciplinary open access archive for the deposit and dissemination of scientific research documents, whether they are published or not. The documents may come from teaching and research institutions in France or abroad, or from public or private research centers.
L'archive ouverte pluridisciplinaire HAL, est destinée au dépôt et à la diffusion de documents scientifiques de niveau recherche, publiés ou non, émanant des établissements d'enseignement et de recherche français ou étrangers, des laboratoires publics ou privés. 
International Journal of Humanoid Robotics

(c) World Scientific Publishing Company

\title{
Active Tactile Transfer Learning for Object Discrimination in an Unstructured Environment using Multimodal Robotic Skin
}

\author{
Mohsen Kaboli, Di Feng, and Gordon Cheng \\ The Institute for Cognitive Systems, Technical University of Munich \\ Arcisstrasse 21 80333, Munich, Germany \\ mohsen.kaboli@tum.de \\ The video to this paper: http://web.ics.ei.tum.de/ mohsen/videos/IJHR2017.mp 4
}

\begin{abstract}
In this paper, we propose a complete probabilistic tactile-based framework to enable a robot with multimodal artificial skin to autonomously explore unknown workspaces and recognize objects via their physical properties while re-using past tactile experience. Our framework consists of three components: (1) an active pre-touch strategy to efficiently explore unknown workspaces; (2) an active tactile learning method to learn about unknown objects based on their physical properties (surface texture, stiffness, and thermal conductivity) by selecting the most informative tactile exploratory action to apply to the object, so that the robot can efficiently distinguish between objects with a small number of actions. (3) an active tactile transfer learning algorithm to enable the robotic system to exploit its prior tactile knowledge to learn about new unknown objects with a few training samples or even one (one-shot tactile learning). Our proposed framework was evaluated using a robotic arm equipped with multimodal artificial skin. The robot with the active pre-touch method reduced the uncertainty of the workspace up to $30 \%$ and $70 \%$ compared to uniform and random strategies respectively. By means of the active tactile learning algorithm, the robot used 50\% fewer exploratory actions than the baseline methods to achieve the same learning accuracy. Taking advantage of the prior tactile knowledge, the robot can discriminate among new unknown objects using only one training sample with a recognition accuracy $10 \%(15 \%)$ higher than the active tactile learning method (the uniform method) without knowledge transfer. Furthermore, the experimental results show that our proposed active tactile transfer learning method is robust against transferring negative prior tactile knowledge.
\end{abstract}

Keywords: Active workspace exploration, active tactile exploration, active tactile transfer learning, pre-touch, tactile sensing, multimodal robotic skin.

\section{Introduction}

Touch plays an important role in our daily lives, from perceiving the environment to identifying, learning about and interacting with objects. It is difficult to compensate for a lack of touch through other senses. For robotic systems that interact with dynamic environments, it is crucial to recognize objects via their physical properties (such as surface texture, stiffness, center of mass, and thermal conductivity). However, this is difficult to achieve even with advanced vision techniques, which are often marred by occlusion, poor lighting situations, and a lack of precision. ${ }^{57,59} \mathrm{As}$ 
an alternative, tactile sensing can simultaneously provide rich and direct feedbacks to the robotic systems from abundant contact points. ${ }^{1}$

We humans use sense of touch to actively explore our environment and objects through their various physical properties such as surface texture, stiffness, shape, and thermal conductivity. To actively learn about and efficiently discriminate among objects based on their physical properties, humans strategically select exploratory actions to perceive objects' properties (e.g. sliding to sense the textural properties, pressing to estimate the stiffness, and static contact to measure the thermal conductivity). Active tactile exploration is a complex procedure which requires efficient perception and learning methods. Furthermore, we intelligently re-use our previously acquired tactile knowledge to actively learn about new objects. Our prior tactile knowledge, or past tactile experience, helps us to efficiently explore new objects by performing a few number of active exploratory actions or just one. In other words, we learn about new objects with a few number of training samples or even one (one-shot learning) while using our prior knowledge. To facilitate these in the robotic system, we propose a robotic active tactile transfer learning method to enable a robotic system to efficiently learn about object's physical properties while exploiting its multiple tactile prior knowledge.

\subsection{Related work}

Haptically accessible object characteristics can be divided into three general classes: geometric information, material properties, and inner properties (e.g. center of mass). ${ }^{52,54}$ Robots can recognize the geometric properties of objects by perceiving their shapes via either proprioceptive receptors ${ }^{2-5}$ or cutaneous receptors, by exhaustively touching a single object with a known orientation and location in the workspace. $^{6-8}$ The object material can be characterized and identified by its textural properties, stiffness, and thermal conductivity. The robot can sense the textural properties of objects using cutaneous tactile receptors by moving fingertips on the objects' surfaces. ${ }^{9-14}$ The stiffness of objects can also be measured by pressing the robot fingertips against the objects. ${ }^{15}$ Likewise, the thermal conductivity can be perceived by making lightly contact with the objects' surfaces. ${ }^{16}$

Previous researchers have used various robotic systems and tactile sensors to passively explore objects via their physical properties and to discriminate among each other by utilizing uniformly collected training samples. ${ }^{17-23}$ They used a predefined number of exploratory movements to sense the physical properties of objects with fixed positions and orientation in a known workspace. Therefore, the autonomy of the robot is limited.

On the contrary, active tactile exploration has shown great potential for enabling the robotic system with more natural and human-like strategies. ${ }^{24}$ An autonomous robot should be able to select and execute the exploratory actions that provide it with the maximum amount of information. In this regard, several approaches were proposed to actively discriminate among objects using their physical properties. 
For instance, $\mathrm{Xu}$ et al..$^{25}$ used the index finger of the Shadow Hand with the BioTac sensor to collect training data by executing three different exploratory actions (pressing for stiffness, sliding for surface texture, and static contact for thermal conductivity) five times on each experimental object. They placed objects under the index finger, and applied a sequence of exploratory movements to construct observation models. The base and wrist of the dexterous robotic hand were fixed on a table, and all joints in the hand and wrist were deactivated (except two joints of the index finger). These physical constraints, therefore, resulted in an approach which is unscalable for robotic tactile exploration. Lepora et al. ${ }^{26}$ controlled a biomimetic fingertip to slide along ten different surfaces to perceive their textural properties. In this work, the measurement of surfaces' positions were noisy. In order to actively discriminate among the surfaces under position uncertainty, the authors constructed the observation models for the textures and the positions of the surfaces offline, by uniformly sampling the collected training data of each surface texture and each possible surface position under a range of contact depths. In another study, ${ }^{27}$ the Weiss Robotics sensor was mounted on the end-effector of a robot arm to classify 21 objects. They created a database of tactile observations offline by grasping each object with a pre-designed trajectory. The authors managed to actively recognize objects using tactile images, which were produced by strategically selecting the height of the robot finger and grasping the objects. Matrins et al ${ }^{28}$ aimed at developing a general active haptic exploration and recognition strategy for heterogeneous surfaces. The experiments were conducted to search and follow the discontinuities between regions of surfaces with two different materials. However, the experiments were only carried out in the simulation using uniformly collected data offline. Tanaka et al. ${ }^{29}$ combined Gaussian process latent variable and nonlinear dimensionality reduction method to actively discriminate among four cups in the real experiments. The authors collected 400 training samples uniformly using three fingers of the Shadow hand, which was fixed and the objects were placed on a turntable. The observation model was constructed with action features using the index finger with 2-DOF to generate inflective and horizontal movements on the objects. Since the proposed method requires a huge amount of training data, the high dimensional action space makes the optimal action search and model learning intractable.

In the above-mentioned work, the location and orientation of the experimental objects in the workspace were known. Moreover, in order to construct the observation models, the training samples were collected uniformly and offline. The informativeness of the training data collected from each object is different. Some objects have distinctive tactile properties, which makes them easy to be discriminated. Therefore, collecting too many training samples by applying exploratory actions is redundant; whereas for objects, whose physical properties are similar and thus can be easily confused with other objects' properties, it is necessary to collect sufficient samples to construct reliable and robust observation models.

Furthermore, collecting training samples are time and memory consuming and 
there may not be always sufficient training data available. In this case, re-using previously obtained tactile knowledge together with a few number of training samples or even one can improve the robotic performance of learning about new objects (tactile transfer learning). Although there are many papers proposing various transfer learning strategies in visual categorization ${ }^{30-37,56,58}$, reinforcement learning, ${ }^{38}$ data mining ${ }^{39-41}$, brain computer interface ${ }^{42}$, and deep learning, ${ }^{43}$ to the best of our knowledge, in the tactile learning domain, it is only our previous work which proposed a tactile transfer learning method for object discrimination (Kaboli et $\left.a l^{44,55}\right)$. In our previous work, we enabled a robotic hands to re-use its learned texture models from the prior objects to discriminate among new in-hand objects via their textural properties with a few training samples or even one. The robotic hand slid its fingers to passively perceive the textural properties of each object at each time and the training samples were collected uniformly across all objects.

\subsection{Contribution}

In this study, we propose a probabilistic tactile-based framework to enable the robotic system with a sense of touch to be one step closer to human tactile exploration and learning. Using our framework, the robot can autonomously explore unknown workspace, actively learn about objects, and efficiently discriminate among new objects while exploiting the prior tactile knowledge. Our contribution is three folds: 1) An active pre-touch approach to enable the robotic systems to autonomously and efficiently explore the unknown workspace in order to determine the number of objects, estimate their locations and orientations; 2) An active tactile learning method to learn about objects based on their physical properties (surface textures, stiffness, and thermal conductivity) with the least possible number of tactile exploratory actions (sliding, pressing, and static contact) to construct reliable prior objects' observation models; 3) An active tactile transfer learning method to enable the robot to autonomously select and exploit its prior tactile knowledge to learn about new objects with a small number of training samples. Our proposed framework is demonstrated in Fig. 2

\section{System Description}

\subsection{Multi-modal Artificial Skin}

In order to emulate a human sense of touch, we have designed and manufactured multi-modal tactile sensors ${ }^{45}$ to provide robotic systems with the ability of pre-touch and sense of touch. Each skin cell has one micro controller and a set of multi-modal tactile sensors, including one proximity sensor, one three-axis accelerometer, one temperature sensor, and three normal-force sensors, (see Table 1). All skin cells are directly connected with each other via bendable and stretchable inter-connectors. 


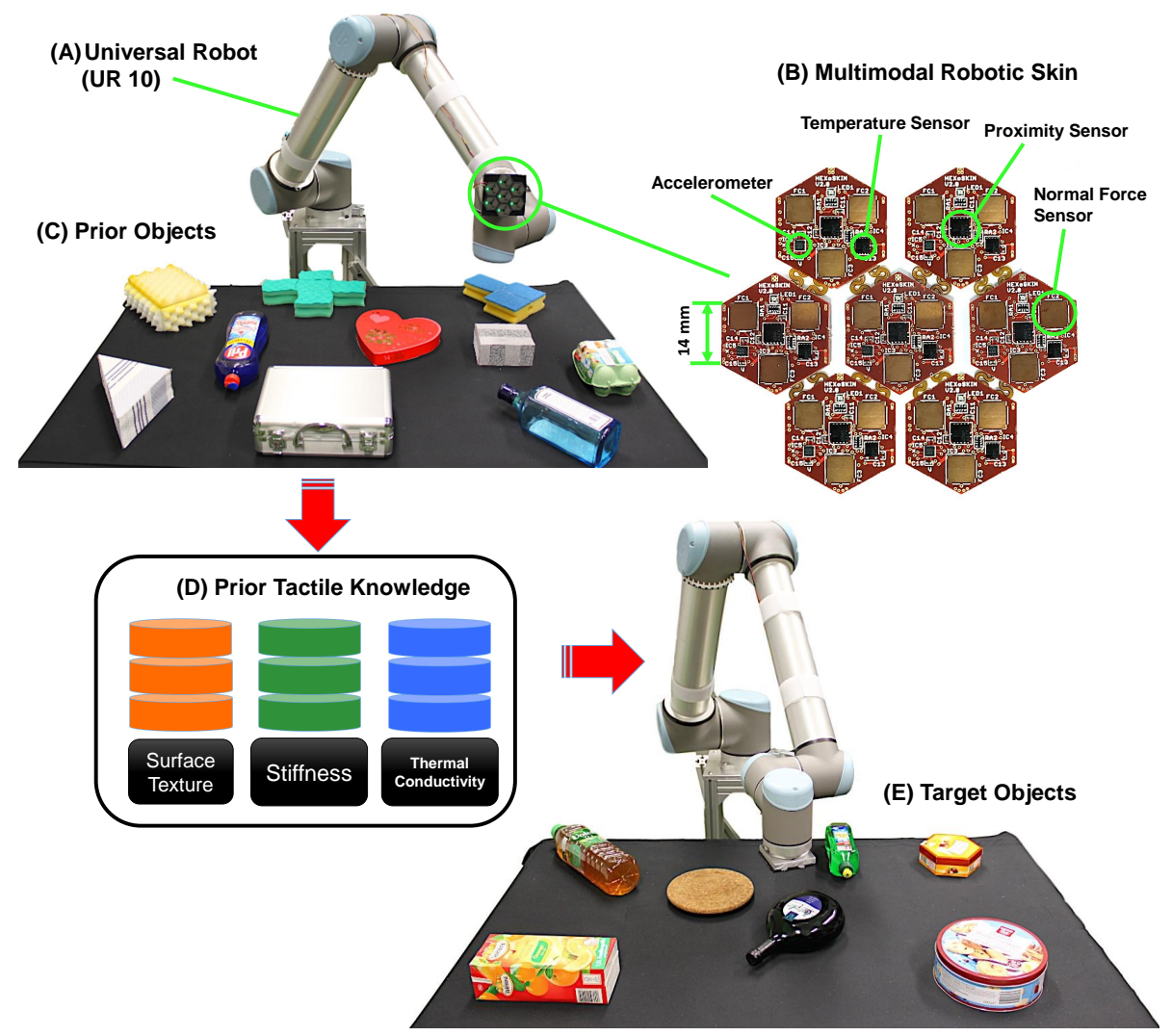

Fig. 1. The scenario of active tactile transfer learning for object discrimination in the unstructured environment. The robotic arm (A) equipped with multimodal artificial skin (B) can actively learn about prior objects $(\mathrm{C})$ in an unstructured environment to build the tactile knowledge of these objects. Then it can leverage this tactile knowledge (D) to actively learn about new objects (E) in another unknown workspace

\subsection{Robotic System}

We mounted one skin patch on the end-effector of the 6-DoF industrial robot called UR10 (Universal Robots). The skin patch consists of 7 skin cells that include: 7 proximity sensors, 7 three-axis accelerometer sensors, 7 temperature sensors, and 21 normal-force sensors (see Fig. 1 (A,B)).

\section{Active Pre-touch for Workspace Exploration}

In this study we use an active pre-touch approach to efficiently explore an unknown workspace. This approach reduces the number of exploratory movements 
Table 1. The multi-modal robotic skin characteristics.

\begin{tabular}{ccccc}
\hline Modality & Acceleration & Force & Proximity & Temperature \\
\hline Sensor & BMA250 & Customized & VCNL4010 & LM71 \\
Per Cell & 1 & 3 & 1 & 1 \\
Range & $\pm 2 g$ & $>0-10 \mathrm{~N}$ & $1-200 \mathrm{~mm}$ & $-40-150^{\circ} \mathrm{C}$ \\
Bandwidth & $0-1 \mathrm{kHz}$ & $0-33 \mathrm{kHz}$ & $0-250 \mathrm{~Hz}$ & $0-7 \mathrm{~Hz}$ \\
\hline
\end{tabular}

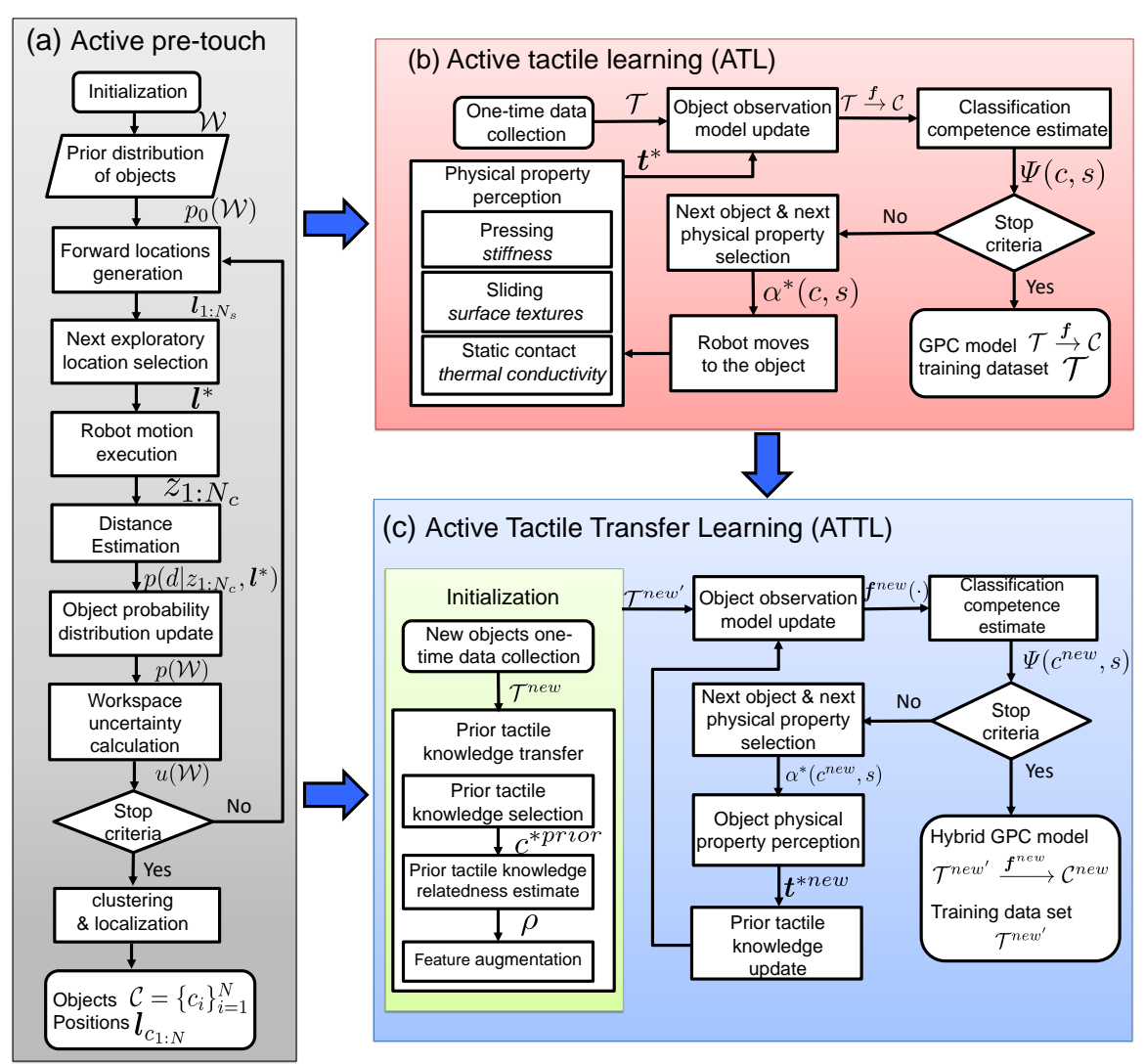

Fig. 2. The proposed framework for active tactile transfer learning for object discrimination in unstructured environment.

and measurements required for unknown workspace exploration. Using this method the robot is able to autonomously estimate the number of objects, their poses, and their geometric centroids.

We consider the workspace $W_{X Y Z} \in \mathbb{Z}^{3}$ as a discretized 3D grid bounded by the reaching capabilities of the robot (see Fig. 3). The artificial skin of the robot has 


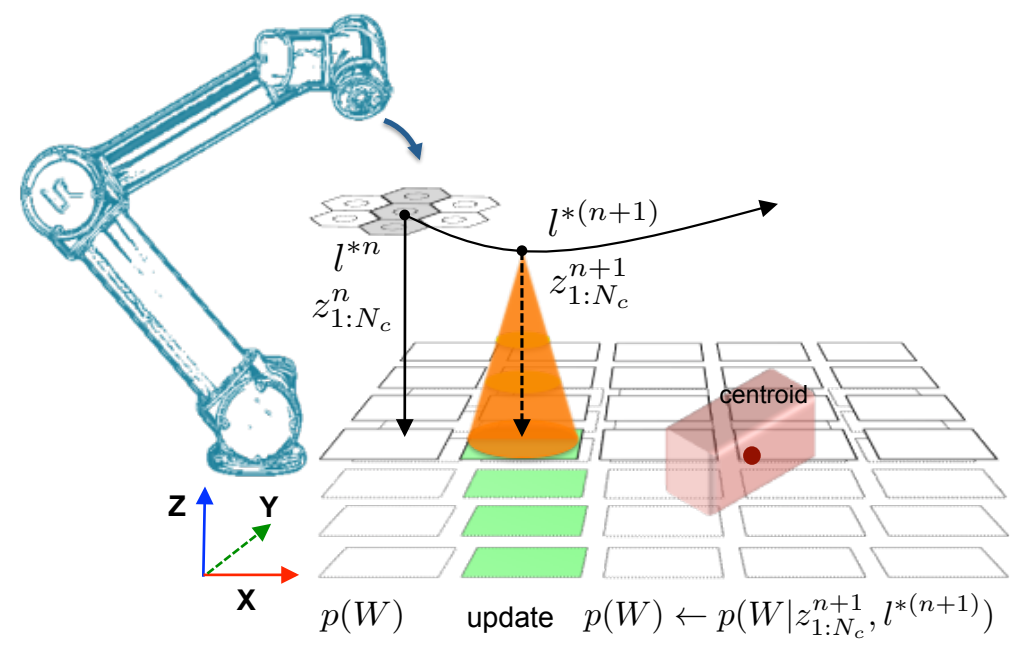

Fig. 3. An illustration of active pre-touch process for unknown workspace exploration.

an array of $N_{c}$ proximity sensors with known locations $\boldsymbol{l}_{1: N_{c}}^{n}$ with respect to the end-effector position $\boldsymbol{l}^{n}$ at each observation $n, \boldsymbol{l}^{n}=\left[x^{n}, y^{n}, z^{n}\right]$. The sensor array outputs a set of measurements $z_{1: N_{c}}^{n}$. To take into account the non-linearities and the uncertainty associated with those measurements, we compute the distance $d$ between the skin cells and the object as the probability $p\left(d \mid z_{i}^{n}, \boldsymbol{l}_{i}^{n}\right)$. By applying Bayes rule we have that $p\left(d \mid z_{i}^{n}, \boldsymbol{l}_{i}^{n}\right) \propto p\left(z_{i}^{n}, \boldsymbol{l}_{i}^{n} \mid d\right) p(d)$. The distribution $p\left(z_{i}^{n}, \boldsymbol{l}_{i}^{n} \mid d\right)$ can be attained by taking proximity sensor measurements $z_{i}$ at different distances $d$. Finally, we define the probability of objects being in the workspace as $p(W)$. We assume that the robot end-effector is horizontal to the X-Y plane of the workspace, therefore the $z$ value in $\boldsymbol{l}$ is fixed.

\subsection{Methodology}

First, the robot starts the exploration from a fixed location corresponding to a grid edge. Then, it generates a set of potential next end-effector locations $\boldsymbol{l}^{n+1} \in L^{n+1}$, i.e., the centre of each neighbouring grid cell. Afterwards, it selects the one that maximizes the probability of detecting objects. The robot performs the next movement and uses the new sensor measurements $z_{1: N_{c}}^{n}$ to update $p(W)$. This process will be iteratively executed until the robot is certain about the workspace, e.g., the entropy of $p(W)$ is below $1 \%$. In order to fuse the measurements taken from all sensor array, we assume that the measurements are independent from each other. The joint probability distribution is given by:

$$
p\left(d \mid z^{n}, \boldsymbol{l}^{n}\right) \propto \prod_{i=1}^{N_{c}} p\left(z_{i}^{n}, \boldsymbol{l}_{i}^{n} \mid d\right) p(d)
$$




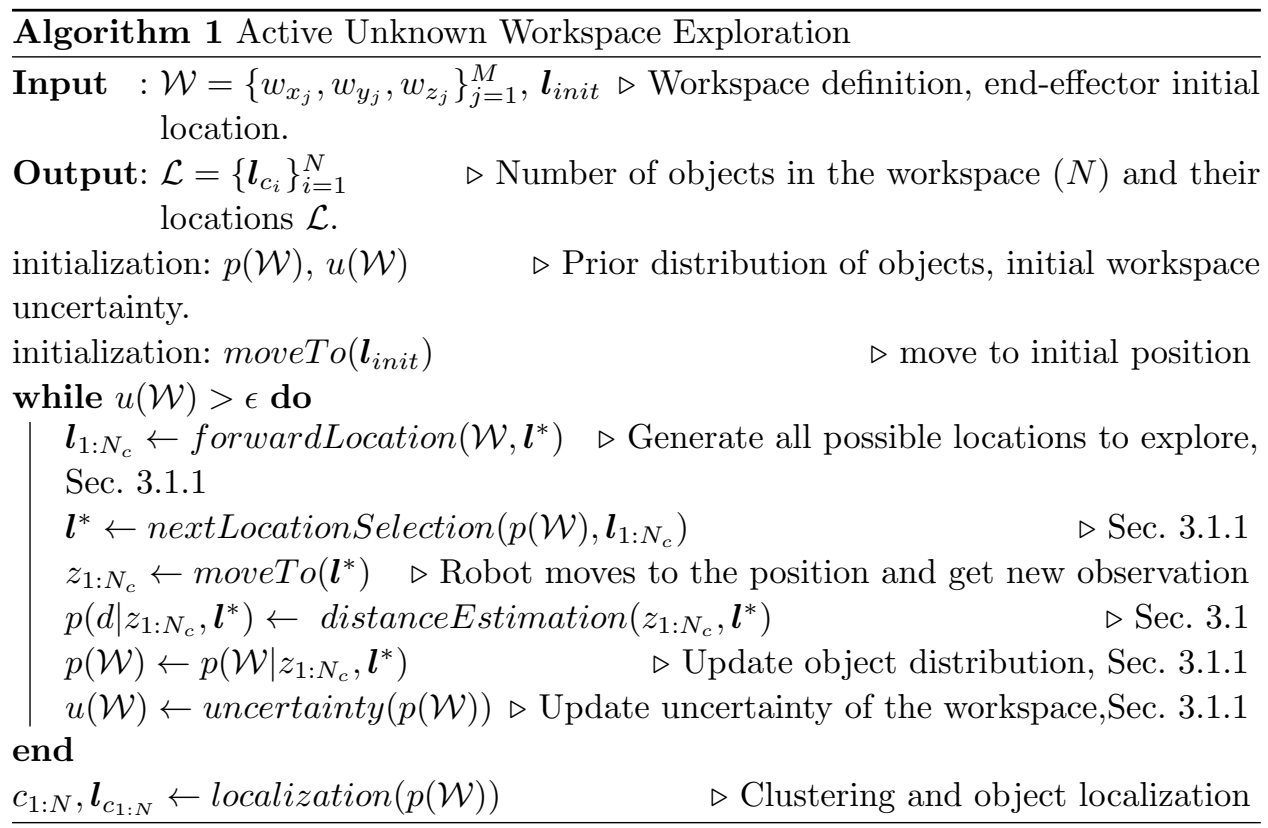

\subsubsection{Next Exploratory Location}

In order to compute the next best location $\boldsymbol{l}^{* n+1}$ of the end-effector, we employ a method based on Lanillos et al. ${ }^{49}$ for air vehicles with radar, which is modified in this study for the proximity sensor array. Considering the current objects estimate in the workspace as $p(W)$, the best next end-effector location is the one that maximizes the probability of detecting objects in the workspace:

$$
\boldsymbol{l}^{* n+1}=\arg \max _{\boldsymbol{l}^{n+1} \in L^{n+1}} \sum_{W} p\left(z_{1: N_{c}}^{n+1}=D \mid \boldsymbol{l}^{n+1}, W\right) p(W)
$$

in which $p\left(z_{1: N_{c}}^{n+1}=D \mid \boldsymbol{l}^{n+1}, W\right)$ is the probability of detecting an object given the next location $\boldsymbol{l}^{n+1}$. Then, $p(W)$ is updated as $p\left(W \mid z_{1: N_{c}}^{n+1}, \boldsymbol{l}^{* n+1}\right)$ (see Fig. 3).

\subsubsection{Objects clustering and centroid estimation}

We estimate the number of objects by a three dimensional connected-labelling algorithm. Then, the 3D minimum bounding box of each object is calculated. It is the smallest enclosing volume that contains all the measurements of the proximity sensors for this object. Finally, the pose and the geometric centroid of each object is computed by means of this bounding box. The proposed active workspace exploration strategy is summarized in Algorithm 1. 

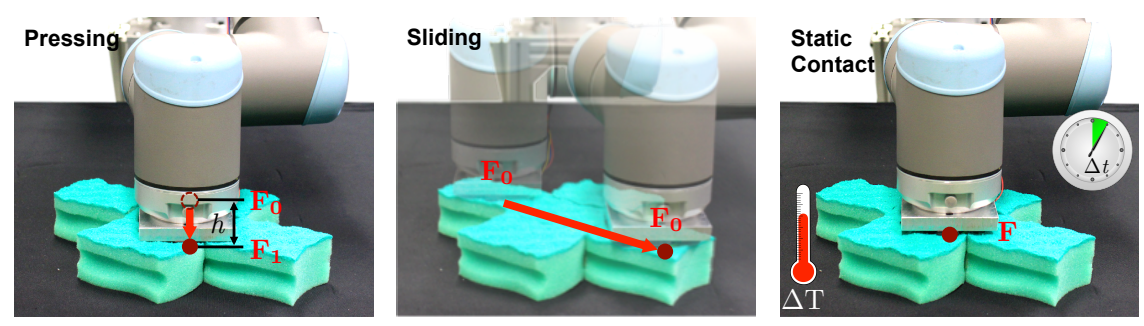

Fig. 4. Exploratory actions: pressing, sliding, and static contact

\section{Object Physical Properties Perception}

A robotic system with the sense of touch needs to execute various exploratory actions on the objects to perceive their physical properties, as we humans do. For instance, a robot slides its sensitive area on the object's surface to sense its textural property, presses on an object to measure its stiffness, and performs static contact to estimate its thermal conductivity.

\section{Stiffness}

The robot measures the stiffness of an object by pressing (see Fig. 4). To do this, the UR10 with the artificial skin on its end-effector first establishes a light contact with the object. The light contact is detected as soon as the measured total normal force $F_{\text {total }}=\frac{1}{N_{c} N_{r}} \sum_{n_{c}=1}^{N_{c}} \sum_{n_{r}=1}^{N_{r}} F_{n_{c}, n_{r}}\left(N_{c}=7\right.$ is the number of skin cell and and $N_{r}=3$ is the number of normal force sensors in each skin cell) exceeds a threshold $f_{\epsilon}$, i.e. $F_{\text {total }}>f_{\epsilon}$. Afterwards, the UR10 presses its sensitive part against the surface of the object. For all normal force sensors, the difference between the force feedbacks $\left(F_{n_{c}, n_{r}}\right)$ recorded before and after pressing $\left(\Delta F_{n_{c}, n_{r}}\right)$ is used as an indication of the object's stiffness in the local contact area. Then the averaged difference value over all force sensors are used to estimate the object's stiffness $\frac{1}{N_{c} N_{r}} \sum_{n_{c}=1}^{N_{c}} \sum_{n_{r}=1}^{N_{r}} \Delta F_{n_{c}, n_{r}}$.

\section{Textural Properties}

To perceive textural properties of objects, the robotic system equipped with artificial skin slides its sensitive part on the surface of objects. In this study, the vibrations (vibro-tactile signals) generated during the sliding movement are measured by the three-axis accelerometer in each skin cell. To extract robust tactile features from the output of each axis of accelerometer sensors $\left(a_{n_{c}}^{x}, a_{n_{c}}^{y}, a_{n_{c}}^{z}\right)$, we previously proposed in Kaboli et al. ${ }^{44}$ a set of tactile feature descriptors $A\left(s_{n}\right), M\left(s_{n}\right)$, and $C\left(s_{n}\right)$ (see Table 2). Moreover, we consider the linear correlations between each axis of the accelerometer $L\left(s_{n}, v_{n}\right)$ as an additional tactile feature. The tactile descriptors for $N_{c}$ number of skin cells are defined as $\mathbf{A}_{\text {total }}, \mathbf{M}_{\text {total }}$, and $\mathbf{C}_{\text {total }}$, and $\mathbf{L}_{\text {total }}$ in Table 2. The total tactile descriptor (TD) is the concatenation of the all computed parameters in one feature vector which can be written as: $\mathbf{T D}=$ 
Table 2. Tactile Feature Descriptors

\begin{tabular}{|l|}
\hline$A\left(s_{n}\right)=\frac{1}{N} \sum_{n=1}^{N}\left(s_{n}-\bar{s}\right)^{2}$ \\
\hline$M\left(s_{n}\right)=\left(A\left(\frac{d s_{n}}{d n}\right) / A\left(s_{n}\right)\right)^{-1 / 2}$ \\
\hline$C\left(s_{n}\right)=M\left(\frac{d s_{n}}{d n}\right) / M\left(s_{n}\right)$ \\
\hline$L\left(s_{n}, v_{n}\right)=\sum_{n=1}^{N}\left(s_{n}-\bar{s}\right)\left(v_{n}-\bar{v}\right) / \sigma\left(s_{n}\right) \sigma\left(v_{n}\right)$ \\
\hline $\mathbf{A}_{\text {total }}=\left[\sum_{n_{c}=1}^{N_{c}} \frac{A\left(a_{n_{c}}^{x}\right)}{N_{c}}, \sum_{n_{c}=1}^{N_{c}} \frac{A\left(a_{n_{c}}^{y}\right)}{N_{c}}, \sum_{n_{c}=1}^{N_{c}} \frac{A\left(a_{n_{c}}^{z}\right)}{N_{c}}\right]$ \\
\hline $\mathbf{M}_{\text {total }}=\left[\sum_{n_{c}=1}^{N_{c}} \frac{M\left(a_{n_{c}}^{x}\right)}{N_{c}}, \sum_{n_{c}=1}^{N_{c}} \frac{M\left(a_{n_{c}}^{y}\right)}{N_{c}}, \sum_{n_{c}=1}^{N_{c}} \frac{M\left(a_{n_{c}}^{z}\right)}{N_{c}}\right]$ \\
\hline $\mathbf{C}_{\text {total }}=\left[\sum_{n_{c}=1}^{N_{c}} \frac{C\left(a_{n_{c}}^{x}\right)}{N_{c}}, \sum_{n_{c}=1}^{N_{c}} \frac{C\left(a_{n_{c}}^{y}\right)}{N_{c}}, \sum_{n_{c}=1}^{N_{c}} \frac{C\left(a_{n_{c}}^{z}\right)}{N_{c}}\right]$ \\
\hline $\mathbf{L}_{\text {total }}=\left[\sum_{n_{c}=1}^{N_{c}} \frac{L\left(a_{n_{c}}^{x}, a_{n_{c}}^{y}\right)}{N_{c}}, \sum_{n_{c}=1}^{N_{c}} \frac{L\left(a_{n_{c}}^{x}, a_{n_{c}}^{z}\right)}{N_{c}}, \sum_{n_{c}=1}^{N_{c}} \frac{L\left(a_{n_{c}}^{y}, a_{n_{c}}^{z}\right)}{N_{c}}\right]$ \\
\hline
\end{tabular}

$\left[\mathbf{A}_{\text {total }}, \mathbf{M}_{\text {total }}, \mathbf{C}_{\text {total }}, L_{\text {total }}\right]$

\section{Thermal Conductivity}

A robotic system with tactile sensing can identify objects through thermal cues by applying static contact on them. When measuring the object's thermal conductivity, the robot contacts its sensitive part with the object surface for a certain time period $t_{\text {contact }}$, during which the average temperature time series of the contacted area is recorded by temperature sensors: $T_{\text {total }}=\left\{\frac{1}{N_{c} N_{T}} \sum_{n_{c}=1}^{N_{c}} \sum_{n_{T}=1}^{N_{T}} T_{n_{c}, n_{T}}^{i}\right\}_{i=1}^{t_{\text {contact }}}$, where $N_{T}$ is the number of temperature sensors in each skin cell, and $T_{n_{c}, n_{T}}$ represents the recordings of a temperature sensor. The final thermal feature (TF) is the combination of the average temperature time series and its gradient $T F=$ $\left[T_{\text {total }}, \Delta T_{\text {total }}\right]$.

\section{Active Tactile Learning (ATL)}

In this section, we describe our proposed probabilistic active tactile object learning method. ${ }^{53}$ Our proposed algorithm enables a robotic system to efficiently learn about objects via their physical properties and to correspondingly construct the observation models of the objects. We start by formalizing the problem. Then, we describe in detail our proposed algorithm called Active Tactile Learning (ATL).

\subsection{Problem Definition}

Consider a scenario in which the robotic system learns about $N$ number of unknown objects $\left(\mathcal{C}=\left\{c_{i}\right\}_{i=1}^{N}\right)$ based on their physical properties (stiffness, surface texture and thermal conductivity) denoted as $\mathcal{S}=\left\{s_{1}, s_{2}, s_{3}\right\}$. These objects are placed in the workspace with the known poses. Some of them may have similar physical 


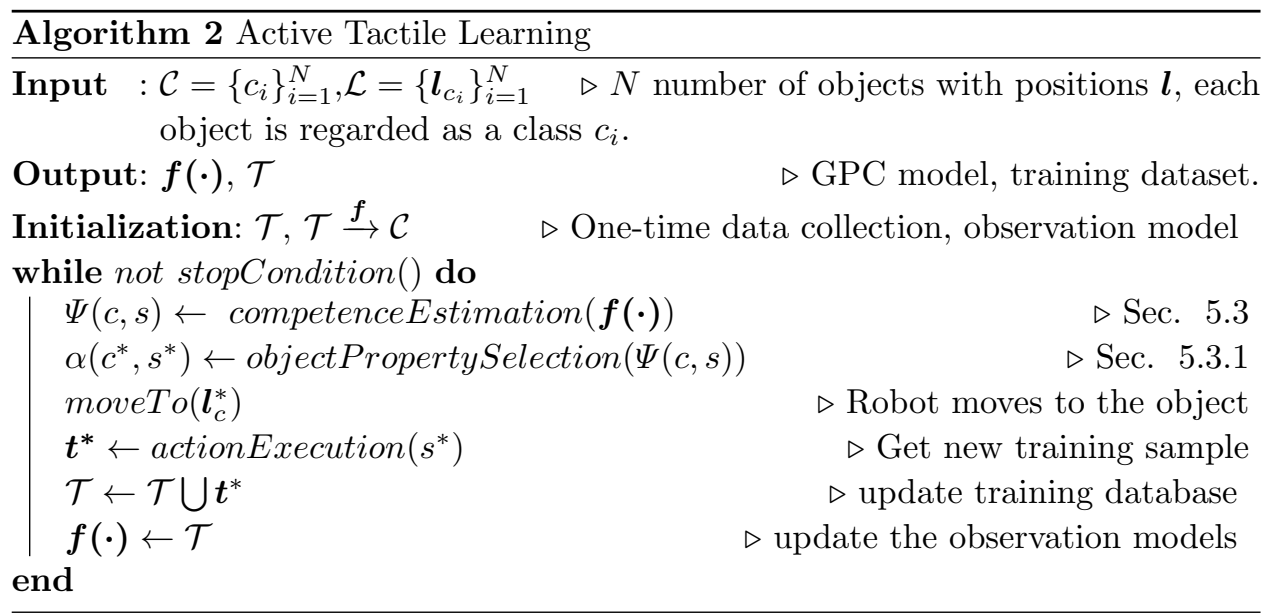

properties, for instance similar surface texture, while some might have quite different properties, for example different stiffness and thermal conductivity. In this scenario, the task of the robot is to efficiently learn about objects by means of their physical properties with as few training samples as possible and to efficiently construct the reliable observation models of the objects. Since the objects with the similar properties cannot be easily distinguished from each other, the robot should autonomously collect more training samples with these objects. The active tactile learning problem is formulated as a standard supervised learning problem for multi-class classification, where each object is regarded as a class; for each tactile property, a probabilistic classifier is efficiently constructed by iteratively selecting the next object to explore and the next physical property to learn, in order to collect the next training sample.

\subsection{Gaussian Process Classification}

In our proposed active tactile transfer learning algorithm, the Gaussian Process Classification (GPC) is employed to construct observation models of the objects. GPC describes the function $\mathcal{X} \stackrel{f}{\rightarrow} \mathcal{Y}$, where $\mathcal{X}$ is the observation set and $\mathcal{Y}$ is the target set which contains integers indicating the labels of the input data. The model assumes that there is an underlying latent function $\mathcal{X} \stackrel{h}{\rightarrow} \mathbb{R}$, which is sampled by the GP prior: ${ }^{50} \boldsymbol{h} \mid \mathcal{X} \sim \mathcal{G P}(\mathbf{0}, \mathcal{K}(\mathcal{X}, \mathcal{X}))$ with zero mean and kernel function $\mathcal{K}: \mathcal{X} \times \mathcal{X} \rightarrow \mathbb{R}$. The kernel function describes the similarity between two observations. The relationship between the label $y_{i}$ and the $\boldsymbol{h}\left(\boldsymbol{x}_{i}\right)$ is then modeled by noise model which can be quite general, such as cumulative Gaussian or sigmoid function, and the inference is achieved by approximation techniques such as Laplace approximation. ${ }^{50}$

In this work, one-vs-all multiclass GPC is employed. For each of the $N$ classes, a binary GPC $\left(\boldsymbol{f}_{n}(\cdot)\right)$ is learned, with its hyper-parameters being optimized through 
maximizing the $\log$ likelihood. ${ }^{50}$ Given a new sample $\boldsymbol{x}^{*}$, each binary classifier predicts the observation probability of its label $p\left(y_{n} \mid \boldsymbol{x}^{*}\right)$. The sample is assigned to the class with the largest predicted probability:

$$
y^{*}=\arg \max _{y_{n} \in \mathcal{Y}} p\left(y_{n} \mid \boldsymbol{x}^{*}\right)
$$

\subsection{Methodology}

The robot starts the learning process by building a training data set (i.e. the feature observations of the objects) with a small number of samples $\left(\mathcal{T}=\left\{\mathcal{T}_{s_{1}}, \mathcal{T}_{s_{2}}, \mathcal{T}_{s_{3}}\right\}\right.$, where $s$ represents a physical property of an object). Then the robot iteratively collects new training data. At each iteration, our algorithm (ATL) updates GPCs with the training data set collected thus far, and estimates the uncertainty in the constructed observation models (classification competence estimation) which guide the robot to the next round of tactile data collection. To do this, the robot first measures the Shannon entropy of each training sample $\boldsymbol{t} \in \mathcal{T}$ :

$$
\mathcal{H}(\boldsymbol{t})=-\sum_{c \in \mathcal{C}} p(c \mid \boldsymbol{t}) \log (p(c \mid \boldsymbol{t}))
$$

Then the training data set of one physical property $\mathcal{T}_{s_{j}}$ will be divided into categories $\mathcal{T}_{s_{j}}=\left\{\mathcal{T}_{s_{j}, c_{i}}\right\}_{i=1}^{N}$, in which $\mathcal{T}_{s_{j}, c_{i}}$ contains $N_{s_{j}, c_{i}}$ number of samples $\left(s_{j}\right.$ denotes the $j$ th physical property and $c_{i}$ represents the $i$ th object). The GPCs competence $\Psi\left(c_{i}, s_{j}\right)$ is estimated as the mean value of the Shannon entropy:

$$
\Psi\left(c_{i}, s_{j}\right)=\frac{1}{N_{s_{j}, c_{i}}} \sum_{\boldsymbol{t}_{s_{j}, c_{i}} \in \mathcal{T}_{s_{j}, c_{i}}} \mathcal{H}\left(\boldsymbol{t}_{s_{j}, c_{i}}\right)
$$

The higher the $\Psi\left(c_{i}, s_{j}\right)$ is, the more uncertain the robot is about the object.

\subsubsection{Next Object and Next Physical Property Selection}

Let us define the object-property pair, $\alpha(c, s)$ as a function of the object $c$ and physical property $s$. After selecting $\alpha(c, s)$, the robot moves to the object $c$ and executes the corresponding exploratory action to perceive the physical property $s$. In order to learn about objects efficiently, the robot can greedily sample the next object and the next property which may bring about the largest improvement to the performance of GPCs (exploitation). Furthermore, to avoid being trapped in the local maxima, we add an exploration rate so that the robot can also randomly select $\alpha(c, s)$ by uniform distribution (exploration). We denote $p_{\alpha}$ as a probability which is uniformly generated with $\mathcal{U}(0,1)$ at each iteration in the active tactile learning procedure. Then the next object $c^{*}$ and next physical property $s^{*}$ is determined by:

$$
\alpha^{*}(c, s)=\left\{\begin{array}{lr}
\underset{c_{i} \in \mathcal{C}, s_{j} \in\left\{s_{1}, s_{2}, s_{3}\right\}}{\arg \max } \Psi\left(c_{i}, s_{j}\right), & \text { if } p_{\alpha}>\epsilon_{\alpha} \\
c=\mathcal{U}\left\{c_{1}, c_{2}, \ldots c_{N}\right\}, s=\mathcal{U}\left\{s_{1}, s_{2}, s_{3}\right\}, & \text { otherwise }
\end{array}\right.
$$


where $\epsilon_{\alpha}$ is the parameter to control the exploration-exploitation trade-off.

The learning process is repeated until a target criteria is reached, in our case, when there is no improvement in the uncertainty of GPCs. Finally, the robotic system constructs reliable observation models $(\mathcal{T} \stackrel{f}{\rightarrow} \mathcal{C})$ and the feature observations of the objects $(\mathcal{T})$ by strategically selecting objects and exploratory actions to perceive the physical properties. The entire process of the ATL algorithm is summarized in Algorithm 2.

\section{Active Tactile Transfer Learning (ATTL)}

The proposed ATL method in Sec. 5 enables the robotic system to efficiently learn about unknown objects via their physical properties by selecting strategically the next object and the next exploratory action. However, the robot is still unable to exploit its prior tactile knowledge when it needs to learn a new set of unknown objects. In this section we propose a method that facilitates the robot to leverage prior tactile knowledge to boost the active learning process for the new unknown objects. We refer to our method as Active Tactile Transfer Learning (ATTL).

\subsection{Problem Definition}

Assume that the robot has exploited the proposed ATL method to learn about $N_{\text {prior }}$ number of unknown prior objects denoted as $\left(\mathcal{C}^{\text {prior }}=\left\{c_{j}^{\text {prior }}\right\}_{j=1}^{N_{\text {prior }}}\right)$ and constructed reliable GPC observation models $\left(\mathcal{T}^{\text {prior }} \stackrel{f^{\text {prior }}}{\longrightarrow} \mathcal{C}^{\text {prior }}\right)$ and feature observations of the prior objects $\left(\mathcal{T}^{\text {prior }}=\left\{\mathcal{T}_{s_{1}}^{\text {prior }}, \mathcal{T}_{s_{2}}^{\text {prior }}, \mathcal{T}_{s_{3}}^{\text {prior }}\right\}\right)$ (Fig. $1(\mathrm{C}, \mathrm{D})$. Now the robot is required to learn a new set of unknown objects (Fig. 1 (E)). We denote $N_{\text {new }}$ number of the new unknown objects as $\mathcal{C}^{\text {new }}=\left\{c_{i}^{\text {new }}\right\}_{i=1}^{N_{\text {new }}}$. Some of the new objects might share similar physical properties with the prior objects (i.e. the new objects and the prior objects are related), while some others might have different physical properties (i.e. the feature observations between new objects and the prior objects are different). The robot is tasked is to actively learn about the new set of objects while re-using its tactile experience from the prior objects that are related to the new objects. In other words, the robot is asked to efficiently build the observation models $\mathcal{T}^{\text {new }} \stackrel{f^{\text {new }}}{\longrightarrow} \mathcal{C}^{\text {new }}$ with the feature observations $\mathcal{T}^{\text {new }}=$ $\left\{\mathcal{T}_{s_{1}}^{\text {new }} \mathcal{T}_{s_{2}}^{\text {new }}, \mathcal{T}_{s_{3}}^{\text {new }}\right\}$ for each physical property of the new objects, while transferring the tactile knowledge of the prior objects which comprises of the GPC observation models $\boldsymbol{f}^{\text {prior }}(\cdot)$ and the feature observations $\mathcal{T}^{\text {prior }}$.

\subsection{Methodology}

Our active tactile transfer learning method has the following four main steps: (1) The robot applies each of the exploratory actions (sliding, pressing, and static contact) one time on each new object, in order to collect a small number of feature observations from the new objects $\mathcal{T}^{\text {new }}$ (one time data collection). (2) For each new 
object and each physical property, the robot transfers the prior tactile knowledge which includes the observation models $\boldsymbol{f}^{\text {prior }}(\cdot)$ and feature observations $\mathcal{T}^{\text {prior }}$. To do this, the robot first determines from where to transfer the feature observations from the prior objects to the new objects (Sec. 6.2.1). Then it uses the selected feature observations and the predictions from the prior objects' observation models to improve the new objects' GPC models (Sec. 6.2.2). (3) Using the strategy from ATL (Sec. 5.3.1), the robot actively selects the next object and next physical property to collect the feature observations of the new objects. (4) After obtaining a new feature observation of the selected physical property, the robotic system updates the prior tactile knowledge for all new objects regarding on only this physical property. This includes reselecting the prior tactile knowledge and transferring it to the new objects (Sec. 6.2.1, 6.2.2). The learning process is repeated until there is no improvement in the uncertainty of the new objects' observation models. Our algorithm is demonstrated by Alg. 3 .

In the sequel, we will explain how the robot selects the prior tactile knowledge (Sec. 6.2.1), and how and how much of this knowledge should be transferred to the new objects (Sec. 6.2.2).

\subsubsection{Prior Tactile Knowledge Selection}

When learning about a new object based on a physical property, we select the most related prior object to transfer its feature observations to a new object (from where to transfer), taking advantage of the prediction from the observation models built by the prior objects. Let $p\left(c_{j}^{\text {prior }} \mid \boldsymbol{t}_{s, c_{i}^{\text {new }}}\right)$ be the prediction of a feature observation of the new object $\boldsymbol{t}_{s, c_{i}^{\text {new }}}$ by the observation model of prior object $c_{j}^{\text {prior }}$ with regard to the physical property $s$. We calculate the average prediction to all the samples that belong to the new object $c_{i}^{\text {new }}$ by $\bar{p}\left(c_{j}^{\text {prior }} \mid \mathcal{T}_{s, c_{i}^{\text {eew }}}^{\text {new }}\right)=\frac{1}{N_{s, c_{i}^{\text {new }}}} \sum p\left(c_{i}^{\text {new }} \mid \boldsymbol{t}_{s, c_{i}^{\text {new }}}\right)$. This value estimates the relatedness of the physical property $s$ between prior object $c_{j}^{\text {prior }}$ and the new object $c_{i}^{\text {new }}$. The higher the value is, the more similar two objects are. Thus, the prior object with the largest average prediction value (denoted as $\left.c_{s, i}^{* \text { prior }}\right)$ can be selected to transfer its feature observations of the physical property $s$ to the new objects :

$$
c_{s, i}^{* \text { prior }}=\underset{c^{\text {prior }} \in \mathcal{C}^{\text {prior }}}{\arg \max } \bar{p}\left(c_{j}^{\text {prior }} \mid \mathcal{T}_{s, c_{i}^{\text {ew }}}^{\text {new }}\right) .
$$

\subsubsection{Prior Tactile Knowledge Transfer}

Sec. 6.2.1 describes from where the robot can transfer the feature observations of the prior objects to the new objects. In this section, we show how and how much the robot can reuse this knowledge. When leveraging the feature observations $\mathcal{T}_{s, j}^{\text {prior }}$ of the physical property $s$ from the prior object $c_{j}^{\text {prior }}$ to the new object $c_{i}^{\text {new }}$, we define $\boldsymbol{h}_{s, j}^{\text {prior }}$ and $\boldsymbol{h}_{s, i}^{\text {new }}$ to be the latent functions of the GPC models built by the 


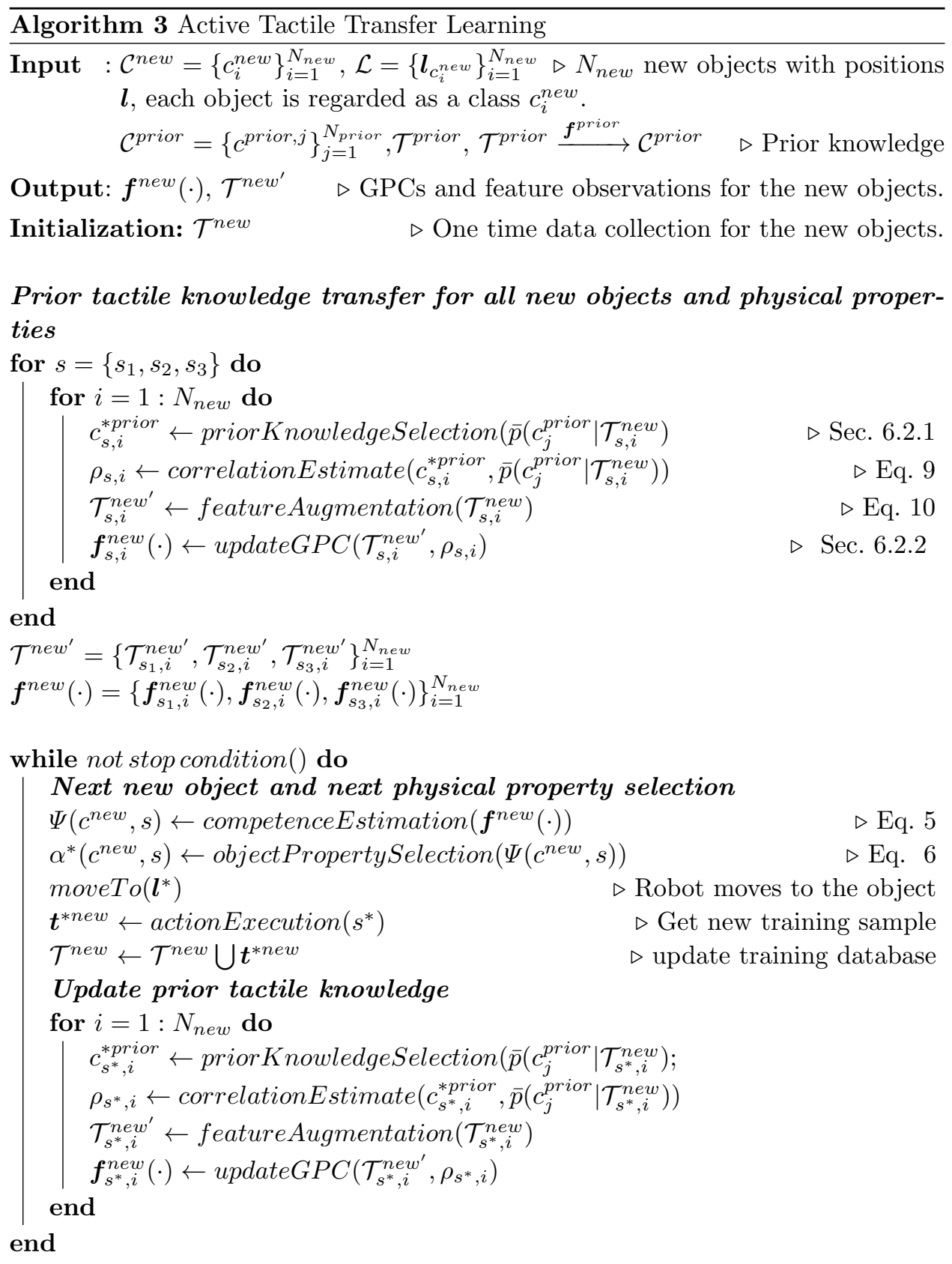

the feature observations from prior objects $\mathcal{T}_{s, j}^{\text {prior }}$ and new object $\mathcal{T}_{s, i}^{\text {new }}$ respectively (Sec. 5.2). It is assumed that these two functions are not independent from each other, but are sampled dependently over a Gaussian prior (hybrid GP). We use this hybrid GP as the observation model of the new object: $\boldsymbol{h}_{s, i}^{\text {new }} \leftarrow\left[\boldsymbol{h}_{s, j}^{\text {prior }}, \boldsymbol{h}_{s, i}^{\text {new }}\right]$. Its 
kernel function is defined as:

$$
\mathcal{K}=\left[\begin{array}{cc}
\mathcal{K}^{t}\left(\mathcal{T}_{j}^{\text {prior }}, \mathcal{T}_{j}^{\text {prior }}\right) & \rho \mathcal{K}^{t}\left(\mathcal{T}_{j}^{\text {prior }}, \mathcal{T}_{i}^{\text {new }}\right) \\
\rho \mathcal{K}^{t}\left(\mathcal{T}_{i}^{\text {new }}, \mathcal{T}_{j}^{\text {prior }}\right) & \mathcal{K}^{t}\left(\mathcal{T}_{i}^{\text {new }}, \mathcal{T}_{i}^{\text {new }}\right)
\end{array}\right]
$$

where $\mathcal{K}^{t}$ is the base kernel function that measures the similarity of training samples. In our case we use radial basis function $(\mathrm{RBF}),{ }^{50}$ whose hyper-parameters are found by maximizing the log-likelihood of this hybrid GPC model.

$\mathcal{K}^{t}\left(\mathcal{T}_{j}^{\text {prior }}, \mathcal{T}_{j}^{\text {prior }}\right)$ and $\mathcal{K}^{t}\left(\mathcal{T}_{i}^{\text {new }}, \mathcal{T}_{i}^{\text {new }}\right)$ measure the similarity for feature observations of the prior object and the new object respectively. $\rho \mathcal{K}^{t}\left(\mathcal{T}_{j}^{\text {prior }}, \mathcal{T}_{i}^{\text {new }}\right)$ and $\rho \mathcal{K}^{t}\left(\mathcal{T}_{i}^{\text {new }}, \mathcal{T}_{j}^{\text {prior }}\right)$ measure the similarity between the feature observations of the prior object and the training samples of the new object. The parameter $\rho$ ranges between 0 and 1 . As analyzed by Chai et al. ${ }^{51} \rho$ controls how much to transfer the feature observations from the prior object to the new object, with $\rho=0$ referring that the prior object and the new object are independent, and little knowledge can be transferred. With $\rho=1$ the two objects are regarded to be the same. We estimate $\rho$ by the average prediction probability of the training samples:

$$
\rho= \begin{cases}\bar{p}\left(c_{j}^{\text {prior }} \mid \mathcal{T}_{s, c_{i}^{\text {ew }}}^{\text {new }}\right), & \text { if } \bar{p}\left(c_{j}^{\text {prior }} \mid \mathcal{T}_{s, c_{i}^{\text {new }}}^{\text {new }}\right)>\epsilon_{\rho} \\ 0, & \text { otherwise }\end{cases}
$$

with $\epsilon_{\rho}$ being the threshold in order to avoid transferring unrelated tactile knowledge.

The method explained above uses the hybrid GP to combine the feature observations of one prior object with a new object. The parameter $\rho$ controls how much to transfer and can stop transferring unrelated tactile knowledge. However, on one hand, $\rho$ only captures the correlation between one prior object and the new object. It cannot weight the influence of the tactile knowledge from the prior object to each training sample which has local variance. On the other hand, the method does not fully exploit all the tactile knowledge from the prior objects, since for each physical property, it selects only one prior object to support a new object. In order to tackle with these issues, we use a simple feature augmentation trick that uses the prediction outputs from all of the observation models of prior objects as auxiliary features to describe the physical property of the objects. The augmented representation of a new sample $\boldsymbol{t}$ is then defined as:

$$
\boldsymbol{t}^{\prime}=[\underbrace{\boldsymbol{t},}_{\text {original feature observation }} \underbrace{p\left(c_{1}^{\text {prior }} \mid \boldsymbol{t}\right), p\left(c_{2}^{\text {prior }} \mid \boldsymbol{t}\right), \ldots, p\left(c_{N_{\text {prior }}^{\text {prior }}} \mid \boldsymbol{t}\right)}_{\text {prior tactile knowledge }}] .
$$

The augmented feature observations are then used to train the hybrid GPC. 


\section{Experimental Results}

\subsection{Experimental Objects}

To evaluate our proposed framework, we deliberately selected two sets of objects, one set with 21 objects as prior objects (Fig. 5) and the other one with 7 objects called new objects (Fig. 6). All experimental objects were made by different materials (such as glass, cardboard, and plastic) with regular and irregular surface textures and various shapes (such as triangular, rectangular, cross, and heart shape). The physical properties of these objects (stiffness, surface textures and thermal conductivity) varied from similar to different.

\subsection{Experimental Setting}

Our proposed tactile-based framework consists of active pre-touch for unknown workspace exploration, active tactile learning (ATL), and active tactile transfer learning (ATTL). To assess in real time the performance of our framework, we designed three experimental scenarios. In the first scenario, the robotic system was asked to autonomously and efficiently learn about the experimental objects (Fig. 5) based on their physical properties (stiffness, surface textures, and thermal conductivity). In the second scenario, the task of the robot was to actively learn about the new set of unknown objects in Fig. 6 by exploiting the tactile experience obtained during the active tactile learning process in Fig. 5. The third scenario was designed to evaluate the robustness of our proposed ATTL algorithm against negative tactile transfer in which the prior objects and new objects had different physical properties. In all three scenarios, the workspace was unknown, and the robot had no knowledge about the number of objects and their positions. It is noteworthy to mention that we arbitrarily changed the light intensity while conducting the experiments, in order to show that our tactile-based framework is robust under different light conditions (Video to this paper: http://web.ics.ei.tum.de/ mohsen/videos/IJHR2017.mp4).

\subsection{Evaluation of Active Tactile Learning (ATL)}

In the first scenario, the robotic system used the proposed active pre-touch method to efficiently explore the unknown workspace. Then, it actively learned about objects by means of their physical properties. Due to the constraint in the workspace, we randomly built 10 groups of objects (each group 10 objects) from Fig. 5 to experiment unknown workspace exploration and active tactile object learning.

\subsubsection{Active Pre-touch for Unknown Workspace Exploration}

Fig. 7(a) illustrates the workspace, which is a cuboid of $110 \mathrm{~cm} \times 64 \mathrm{~cm} \times 10 \mathrm{~cm}(\mathrm{~L} \times$ $W \times H)$. A corresponding Cartesian coordinate frame (world coordinate frame) was defined along its length edge (X-axis), width edge (Y-axis), and height edge (Z-axis). This workspace was discretized into $27 \times 24 \times 10$ grid cells. During the exploration, 


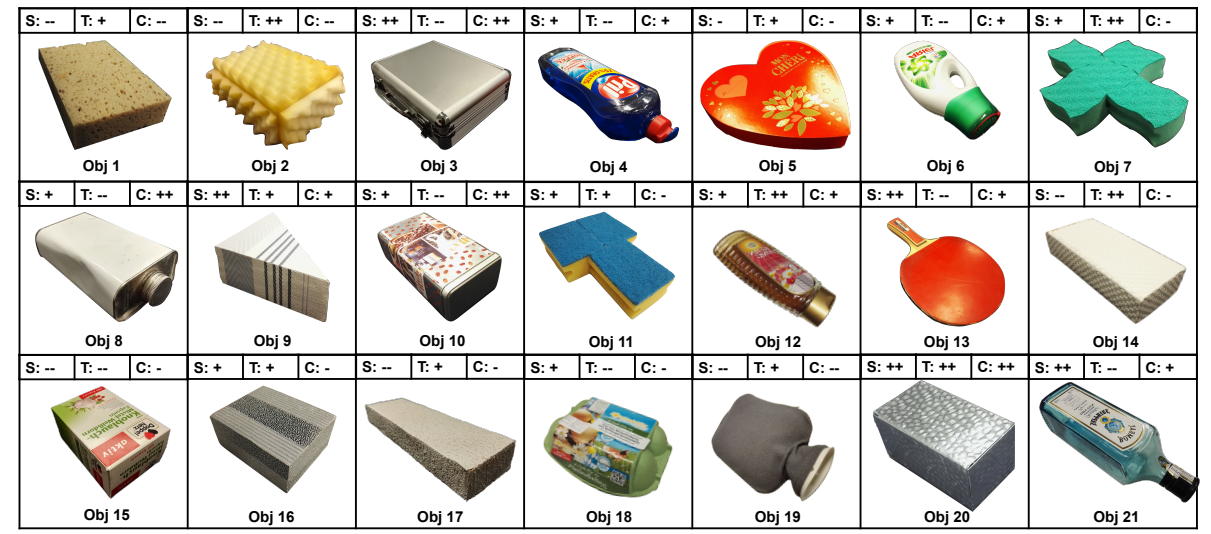

Fig. 5. Prior objects. The physical properties are evaluated subjectively by human subjects and are indicated in the panel to the upper right of each object (S: stiffness, T: roughness of surface textures, C: thermal conductivity. ++: very high; +: high; -:low; -: very low.)

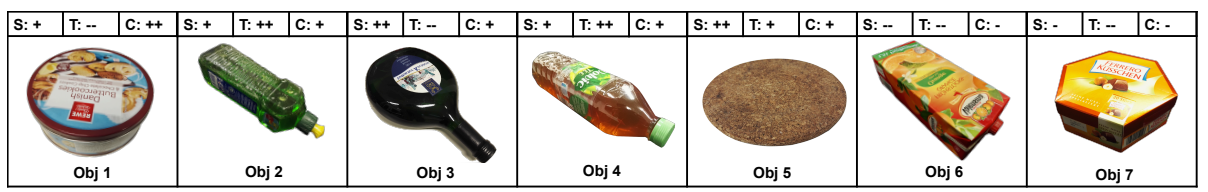

Fig. 6. New objects.

the sensor array (the end-effector of the robot) was positioned at the maximum height of the workspace and horizontal to the X-Y plane. The performance of our proposed active pre-touch method was compared with the random and uniform strategies which served as baseline. We calculated the grid entropy to measure the uncertainty of the workspace during the exploration. The experiment was run 10 times. In each trial, the maximum number of the robotic movements was set to 600. Fig. 7 (e) illustrates that using the proposed active pre-touch strategy, the robot significantly reduced its uncertainty in the workspace compared to random and uniform approaches. This is due to the fact that with our proposed method, the robot explored the locations with a higher probability of observing an object. Fig. 7(b)-7(d) illustrates the results of workspace exploration after 300 steps. Fig. 7 (d) shows that using our active pre-touch strategy, all of the ten objects were successfully clustered and localized, whereas random and uniform strategies suffered from insufficient exploration of the workspace, yielding either wrong determination of the objects' number or wrong geometry estimation (Fig. 7(b) and 7(c)). 


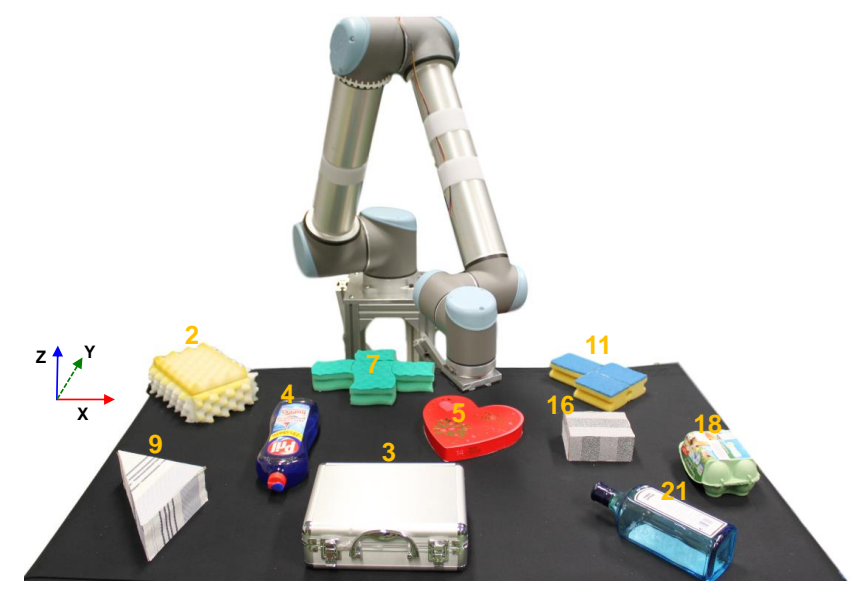

(a) The unknown workspace

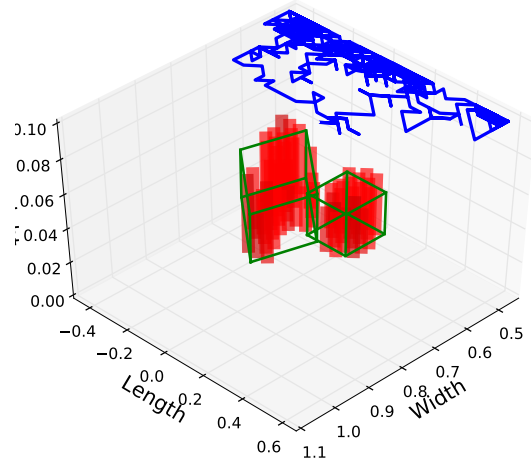

(b) Random

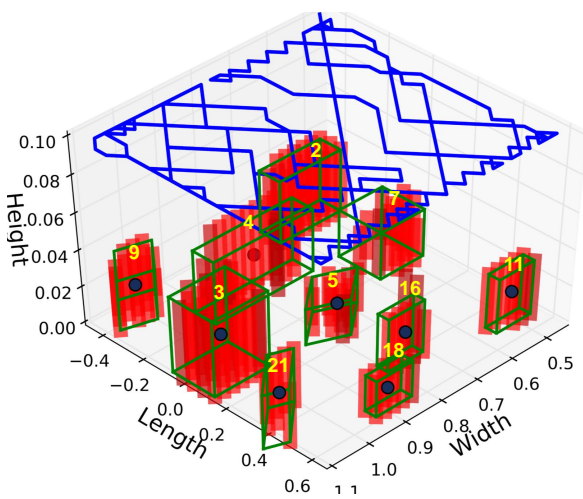

(d) Active pre-touch

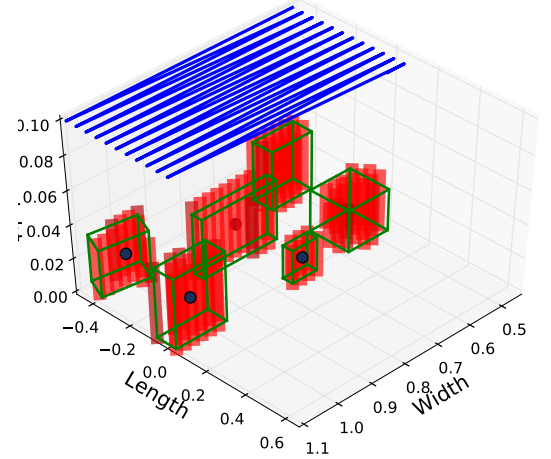

(c) Uniform

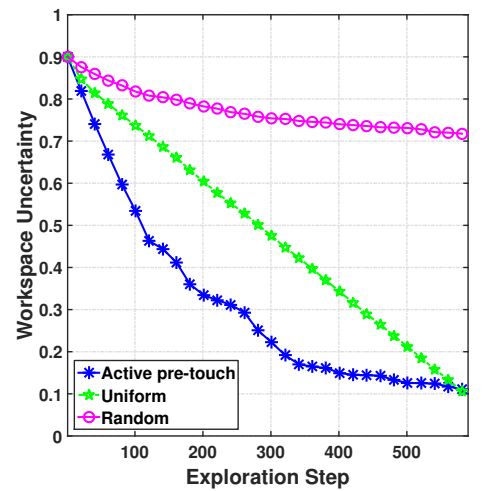

(e) Comparison

Fig. 7. (a): The unknown workspace which the robot explored. (b)-(d): Trajectories of the robot's end-effector during the exploration of the workspace and the localization results using three methods (b) Random strategy. (c) Uniform strategy. (d) Active pre-touch strategy. (e): Statistical evaluation of the active pre-touch method compared with random and uniform strategies. The horizontal axis shows the number of movements the robot has when exploring the workspace. The vertical axis shows the uncertainty in the workspace calculated by the Shannon entropy. 


\subsubsection{Test Data Collection}

The proposed active tactile learning (ATL) was evaluated with a test database constructed automatically by the robot by performing the three exploratory movements (pressing, sliding, and static contact) 20 trials on each experimental prior objects (Fig. 5). The robot begun to apply each of the exploratory movement with a light contact with objects with approximately $0.05 \mathrm{~N}$ of the total normal force measured by the artificial skin. For the pressing movement, the robot first pressed the cellular skin $2 \mathrm{~mm}$ on the objects' surface and then recorded the normal force feedback for $3 s$. To perceive the surface texture of the objects, the robotic system slid the cellular skin on the objects with constant velocity of $1 \mathrm{~cm} / \mathrm{s}$ for $3 \mathrm{~s}$. When measuring the thermal conductivity, the robot pressed its sensitive part $2 \mathrm{~mm}$ on the objects' surface and hold for $15 \mathrm{~s}$. Then it raised its end-effector for $30 \mathrm{~s}$ so that the temperature sensor recovers to ambient temperature. In this way the robot could measure the temperature change during the static contact with a similar initial temperature condition.

\subsubsection{Active Tactile Learning via Three Physical Properties}

To initialize the active tactile learning process, the robot collected small training samples by performing each of three exploratory actions once on each object in the workspace see (Fig. 1 (C) and Fig. 5). Each step when the robot sampled a new training instance, the recognition accuracy of GPCs was measured with the test data set. The performance of ATL was compared with the random and uniform strategy. In this regard, for each group of 10 unknown objects, the experiment was run 30 times using each approach. Fig. 8(a) shows recognition accuracy averaged over all trials. ATL consistently outperforms the other methods by obtaining the same recognition accuracy with fewer performed exploratory actions or training samples. For instance, the robot had in average $50 \%$ fewer training samples compared to the other methods, when it attained the recognition accuracy of $55 \%$ (Fig. 8(a)). Therefore, the robot following ATL method can efficiently construct reliable observation models of object physical properties.

\subsubsection{Active Tactile Learning via One Physical Property}

In order to assess further the performance of the ATL algorithm, the robot was additionally asked to learn about objects in Fig. 5 via one of the three tactile properties individually. Fig. 8(b), 8(c), and 8(d) indicate that the learning progress was dependent on the distributions of the extracted features of the physical properties. Fig. 8(b) shows that learning about objects via their stiffness is more difficult, whereas the learning process for object surface texture and thermal conductivity were faster and resulted in higher recognition accuracy (Fig 8(c) and 8(d)). In all three cases, ATL performs better than random and uniform methods. It can be concluded that using our proposed method (ATL), the robot can efficiently learn 


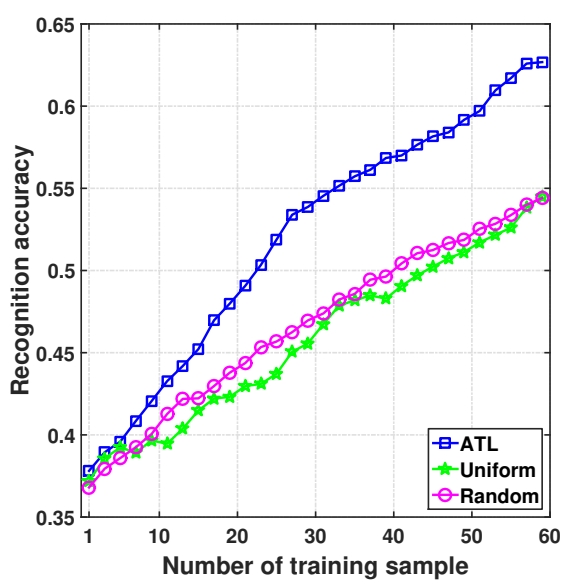

(a) Three physical properties

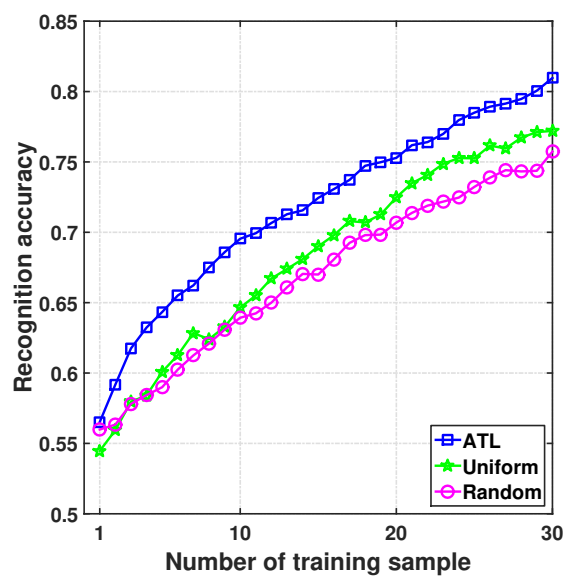

(c) Surface texture

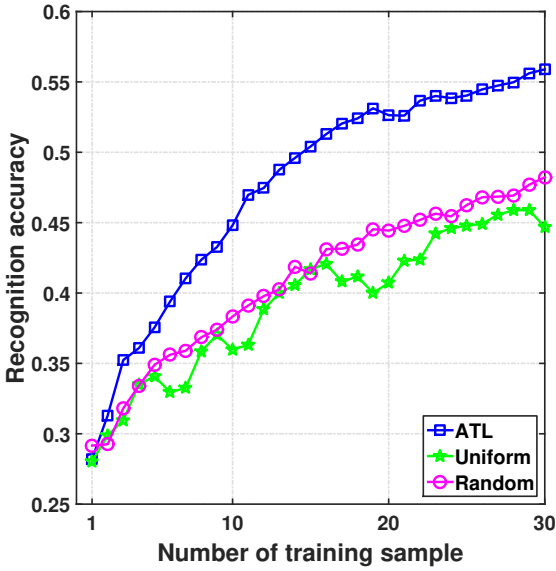

(b) Stiffness

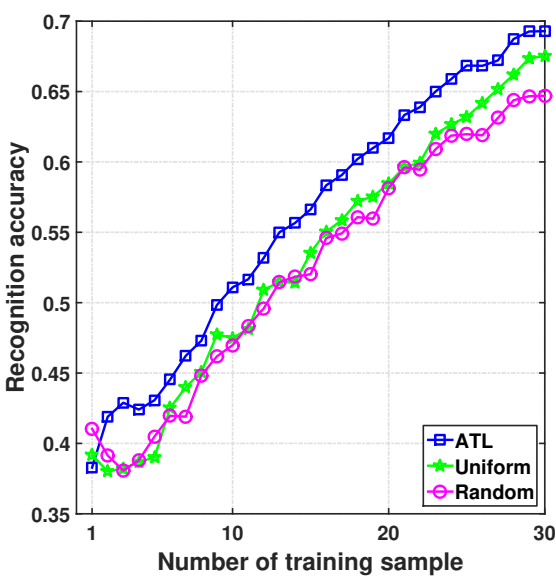

(d) Thermal conductivity

Fig. 8. (a): Evaluation of ATL with three physical properties (stiffness, surface textures, and thermal conductivity). (b)-(d): Evaluation of ATL by learning about the experimental objects via one physical property (b): Stiffness. (c) Surface textures. (d) Thermal conductivity. The horizontal axis represents the learning steps (at each step the robot applied an exploratory action to collect a new training sample), and the vertical axis represents the value of average recognition accuracy on the test dataset.

about objects even with one modality of its artificial skin.

\subsection{Evaluation of Active Tactile Transfer Learning}

In this experiment, we evaluated our proposed active tactile transfer learning strategy for new objects (Fig. 6) using the tactile knowledge constructed efficiently in 
the previous scenario with the prior objects shown in Fig. 5. In order to localize objects and estimate their geometric information, the robot actively explored the unknown workspace following the same procedure as described in Sec. 7.3.1.

\subsubsection{Test Data Collection}

The performance of active tactile transfer learning (ATTL) was assessed with a test database constructed by the robot with the new objects shown in Fig. 6 following the procedure described in Sec. 7.3.2.

\subsubsection{Learning about New Objects with Ten Prior Objects}

To initialize the active tactile learning process, the robot collected one training sample for each new object and each physical property (stiffness, surface texture, and thermal conductivity) in Fig. 1. Then the collected training samples together with the feature observations from the most relevant prior objects were used to construct hybrid observation models via ATTL. At each step when the robot sampled a new training instance, the new hybrid observation models were trained with all the training samples collected hitherto and the updated prior tactile knowledge. The object recognition accuracy was measured with the test data set. The performance of our proposed method was compared with the ATL method and the uniform method which served as the baseline. Using the ATL method, although the robot could efficiently select the next object and the next physical property to learn, it could not re-use its past tactile experience. Using the uniform approach, the robot uniformly collected the training samples for the new objects and physical properties without transferring the prior tactile knowledge (Fig. 1).

The experiment was conducted with ten groups of randomly selected prior objects (each group consists of ten objects) that were used to construct prior tactile knowledge in order to learn about new objects. For each group of prior objects, the experiment was repeated 30 times. In each trial, the robot collected 60 training samples in total using different approaches, thus we had a fair comparison between ATTL and the baseline methods. Fig. 9(a) illustrates that ATTL method consistently outperforms the ATL and uniform methods by reaching higher recognition accuracy with same training samples. For instance, the robot had in average $15 \%$ and $20 \%$ higher recognition accuracy than the ATL and uniform strategies respectively, when only one training sample was collected (one-shot learning) (Fig. 9(a)). By increasing the number of training samples from 1 to 60, the robotic system using the ATTL method leveraged the past tactile experience and achieved $80 \%$ recognition accuracy, whereas following ATL and uniform methods the recognition accuracy reached $75 \%$ and $70 \%$ respectively. We also evaluated ATTL when the robot used only one of the physical properties (stiffness, surface texture and thermal conductivity) to learn about new objects. In this instance, the total number of training samples the robot collected was set to 30. Fig. 9(b), Fig. 9(c) and Fig. 9(d) 
show that in all three cases, ATTL outperforms ATL and uniform strategies. Therefore, using ATTL the robot can efficiently construct reliable observation models of new objects with fewer training samples, when learning about these objects based on either three physical properties or only one property.

\subsubsection{Decreasing the Number of Prior Knowledge}

In this experiment, five out of the 21 experimental objects (Fig. 5) were randomly selected as prior objects. Then the same experimental process explained above was repeated with these five prior objects. Fig. 10(a) illustrates the averaged recognition rate when the robot learned about new objects based on all three physical properties. Fig. 10(b), Fig. 10(c), and Fig. 10(d) show the results with only one of the three physical properties. The results show that the ATTL method that exploited the prior tactile knowledge outperforms both ATL and uniform methods. Comparing Fig. 10(a) with Fig. 9(a), it can be seen that the robotic system achieved lower recognition rate to discriminate among new objects when it used fewer prior objects. This is due to the fact that reducing the number of prior objects decreases the probability of finding highly relevant prior tactile knowledge for the new objects.

\subsection{Consistency Evaluation of ATTL for Negative Tactile Knowledge Transfer}

In transfer learning, the constructed prior knowledge is not always relevant to new tactile observation models. In this case, a brute-force transfer may even degrade the learning performance, generating a so called negative knowledge transfer. When the new and the prior objects are not a good match, a transfer learning method should avoid leveraging negative knowledge.

In this experiment, we evaluated our proposed active tactile transfer learning algorithm against the negative tactile knowledge transfer. To do this, we constructed confusion matrices for all 28 experimental objects w.r.t each physical property in order to find out which of the prior objects were similar and dissimilar to the new objects. The confusion matrices were constructed by training the Support Vector Machine (SVM) models for all 28 objects with ten training samples randomly selected for each object, and using the trained SVM to predict ten unobserved data instances. We calculated the average confusion between objects and normalized the values between 0 and 1, with 0 being totally dissimilar and 1 highly similar. Fig. 7.4.3, Fig. 7.4.3, and Fig. 7.4.3 demonstrate the resulting confusion matrices constructed for stiffness, texture, and thermal conductivity respectively. The blue index indicates the prior objects, and the red index new objects. Regarding stiffness, the prior objects $\{1,2,3,9,13\}$ were totally unrelated to the new objects; for surface texture, prior objects $\{6,7,9,10,21\}$; and for thermal conductivity, prior objects $\{4,6,8,10,13\}$. Therefore, we respectively selected these objects to construct prior tactile knowledge and test ATTL performance, when the robot learned about new objects based on each physical property. We also used objects $\{2,3,6,10,13\}$ as 


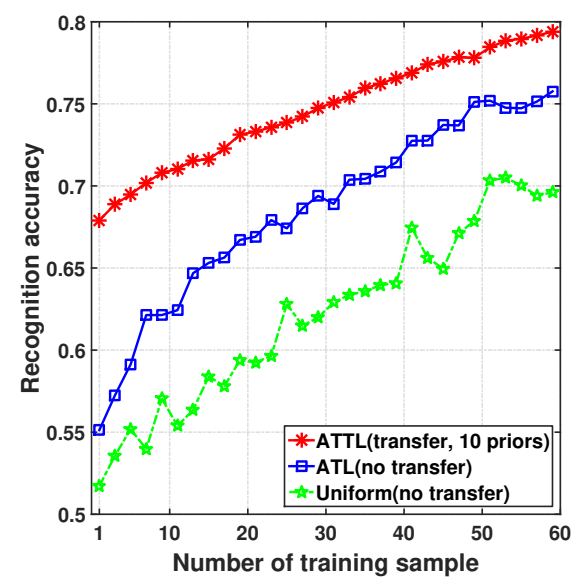

(a) Three physical properties

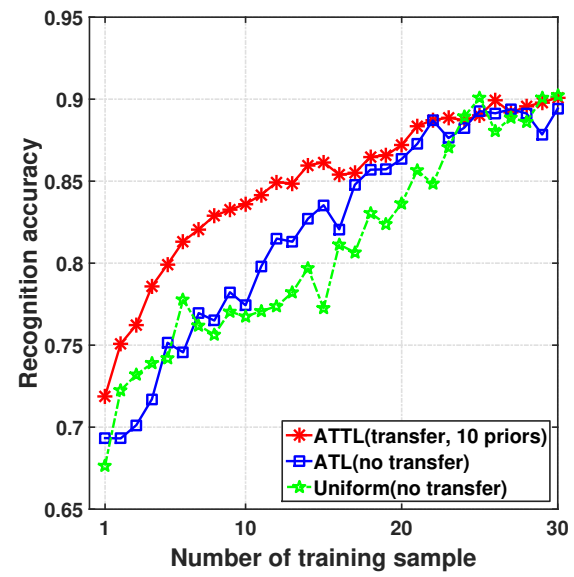

(c) Surface texture

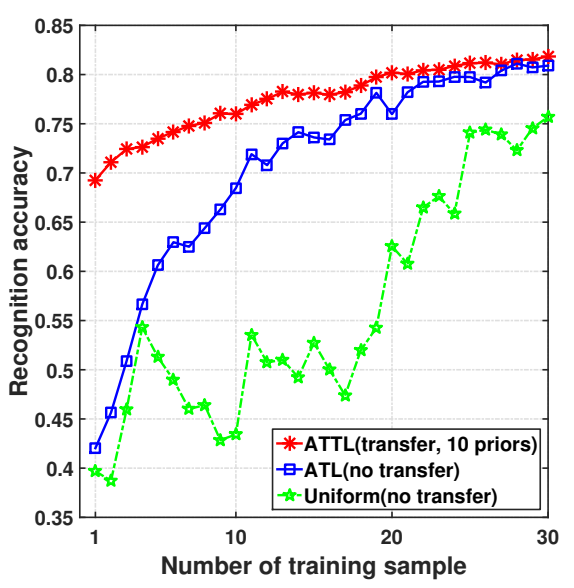

(b) Stiffness

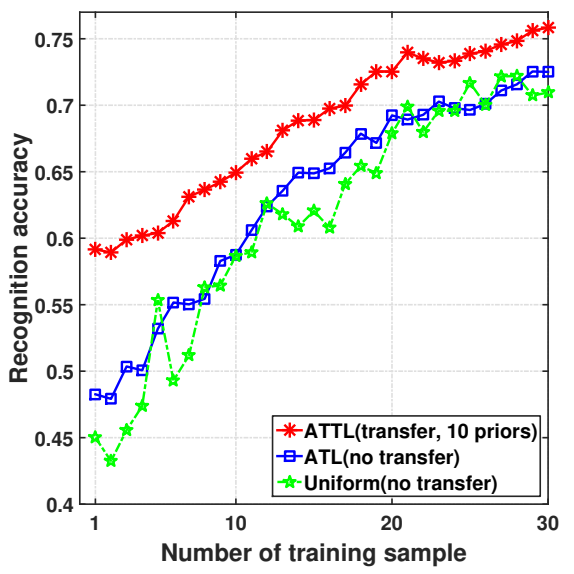

(d) Thermal conductivity

Fig. 9. Evaluation of the active tactile learning performance using ten prior objects. ATTL is compared with the ATL(no transfer) and uniform (no transfer) methods. The horizontal axis represents the growing number of training samples, and the vertical axis represents the averaged value of recognition accuracy on the test data. (a) Learning about the new objects based on three physical properties; (b) based on only stiffness; (c) based on only surface texture; (d) based on only thermal conductivity.

prior objects for learning based on three properties. The performance of the ATTL method was compared with ATL which served as the baseline. The rest of the procedure was similar to Sec. 7.4.

Fig. 15 illustrates the recognition performance attained using ATTL and ATL (no transfer). The results show that the recognition performance achieved by ATTL with irrelevant prior objects is similar to the ones obtained with the ATL 


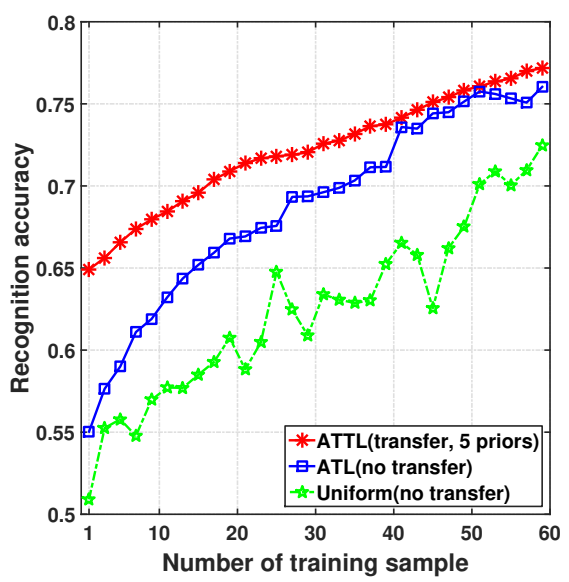

(a) Three physical properties

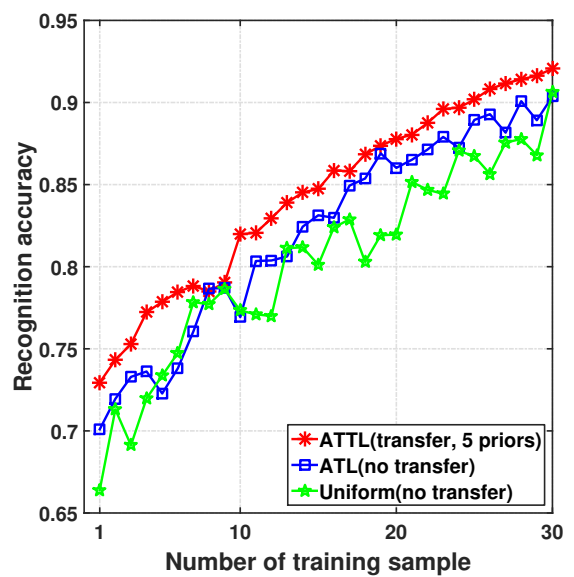

(c) Surface texture

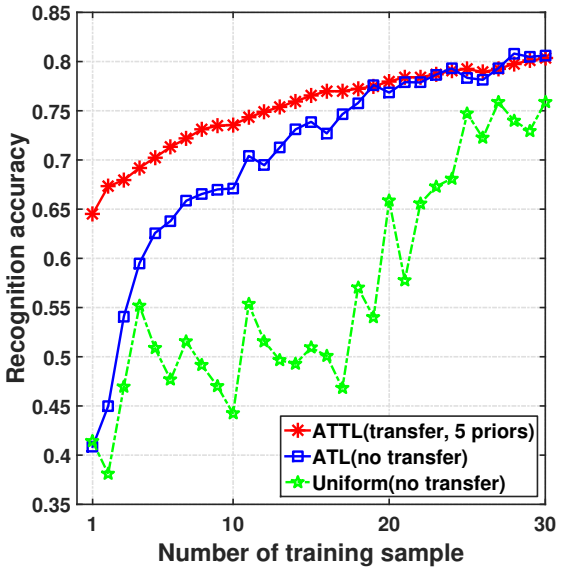

(b) Stiffness

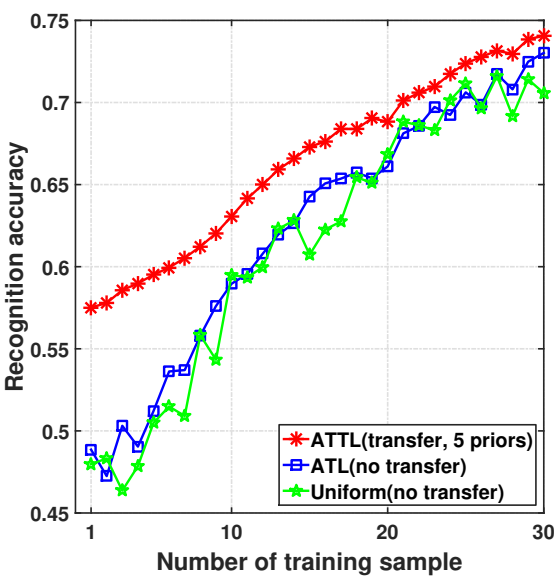

(d) Thermal conductivity

Fig. 10. Evaluation of the ATTL performance using five prior objects. (a) Learning about the new objects based on three physical properties; (b) based on only stiffness; (c) based on only surface texture; (d) based on only thermal conductivity.

method (no-transfer) in the case of learning about objects via three physical properties (Fig. 15(a)) and via only one physical property (Fig. 15(b), Fig. 15(c), and Fig. 15(d)). This indicates that our proposed ATTL can stop transferring irrelevant prior knowledge.

\section{Discussion}

We proposed a framework for a robotic system with multi-modal artificial skin that consists of three parts: active pre-touch, active tactile learning (ATL), and active 


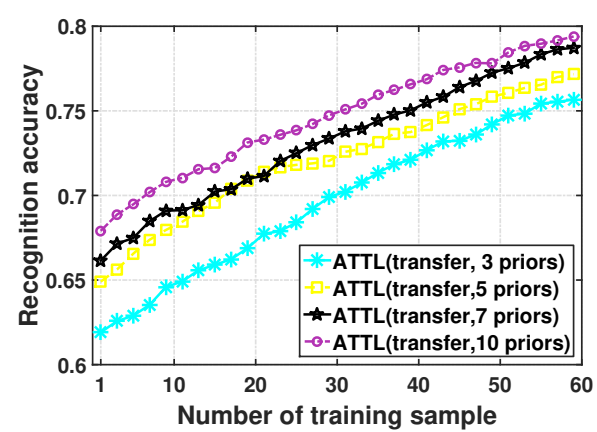

(a) Three physical properties

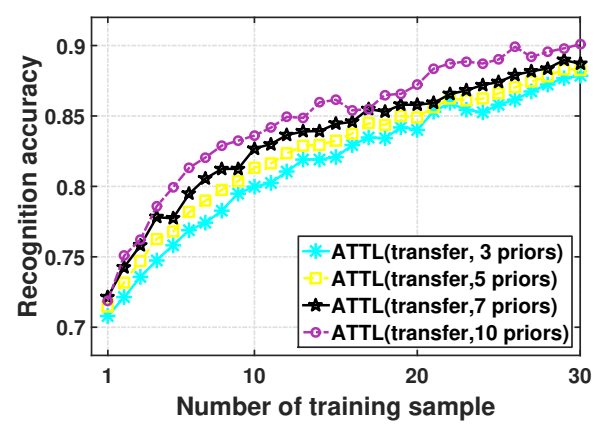

(c) Surface texture

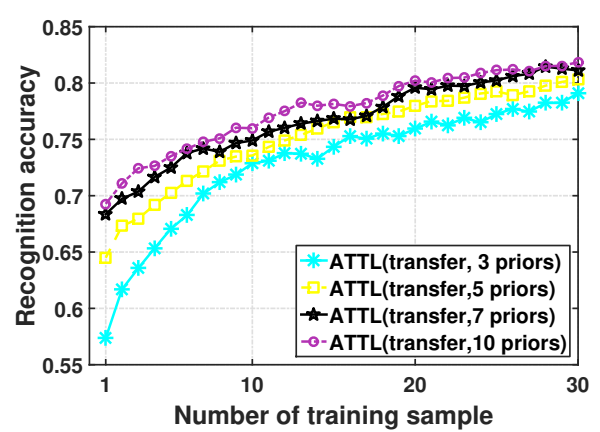

(b) Stiffness

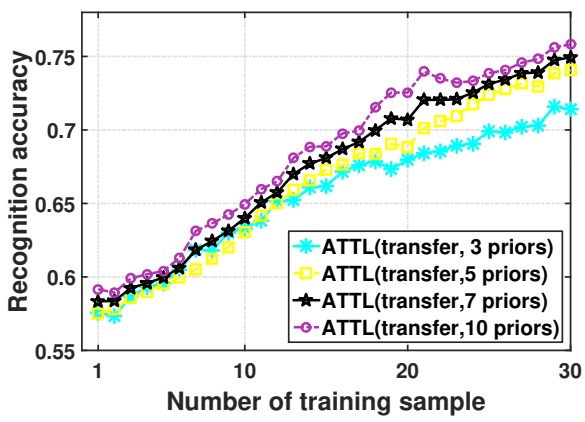

(d) Thermal conductivity

Fig. 11. Evaluation of the ATTL performance using different number of prior objects. (a) Learning about the new objects based on three physical properties; (b) based on only stiffness; (c) based on only surface texture; (d) based on only thermal conductivity.

tactile transfer learning (ATTL) .

Taking advantage of our proposed pre-touch exploration approach, the robotic system can strategically select the next exploratory location in the workspace to efficiently collect pre-touch information. The attained data were then clustered to ascertain the number of objects in the workspace. The minimum bounding box was calculated for each cluster to estimate the object location, orientation, and geometric center. The experimental results show that our proposed method outperforms both random and uniform approaches.

By employing the proposed active tactile learning method (ATL), the robot efficiently learnt about the objects via their physical properties (surface texture, stiffness, and thermal conductivity). It strategically selected the next object and applied the most informative exploratory actions (sliding, pressing, static contact) to attain its feature observations. In the previous studies, the observation models were constructed with the training samples collected uniformly and offline. As the number of training samples grew, the computation became costly. In this case, a 


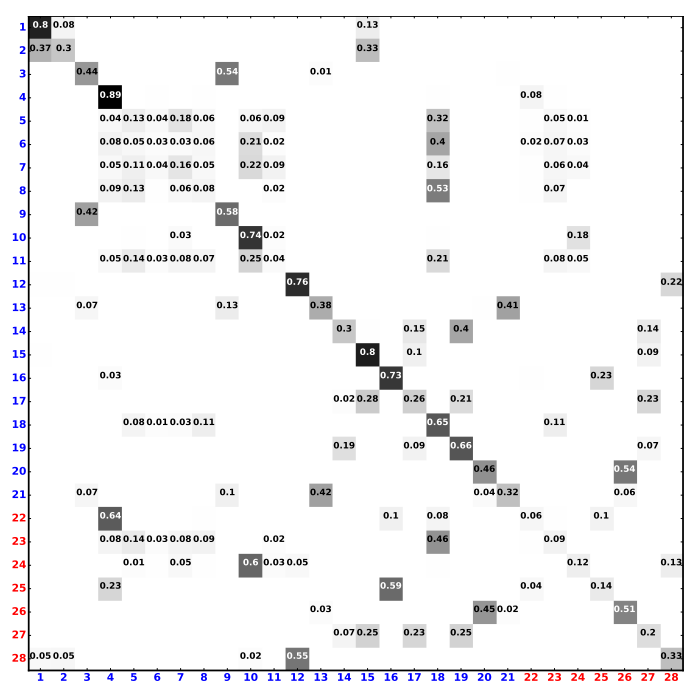

Fig. 12. Confusion matrix for stiffness of 28 objects (prior objects (from 1 till 21 ) + new objects (22 till 28)).

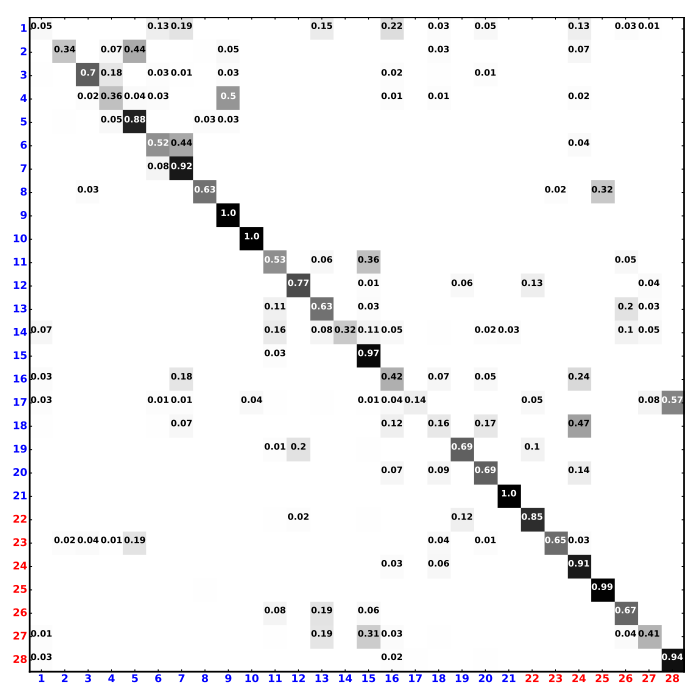

Fig. 13. Confusion matrix for surface texture of 28 objects (prior objects (from 1 till 21) + new objects (22 till 28)).

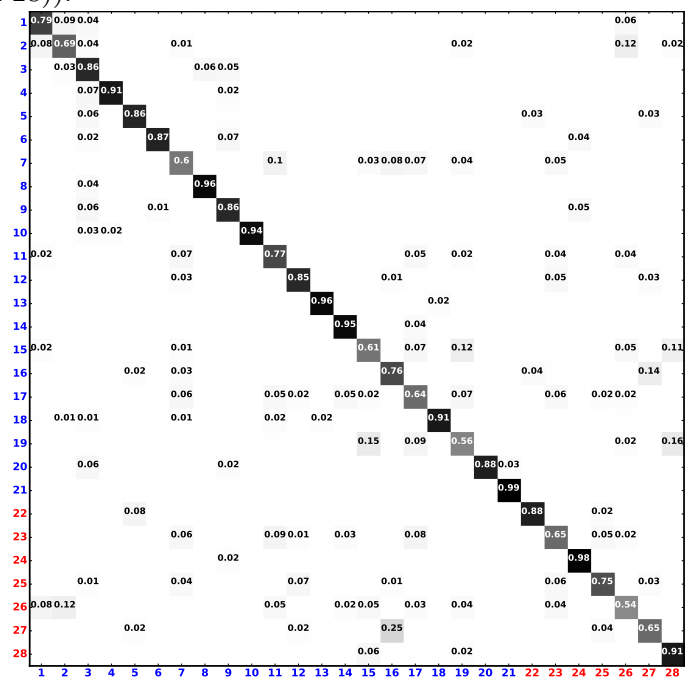

Fig. 14. Confusion matrix for thermal conductivity of 28 objects (prior objects (from 1 till 21) + new objects $(22$ till 28)). 


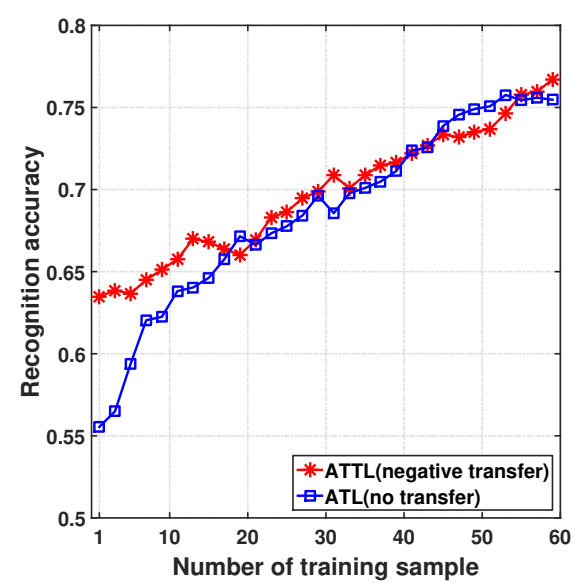

(a) Three physical properties

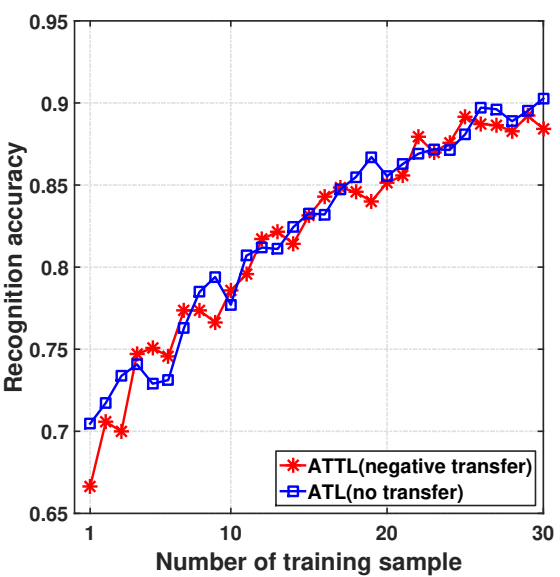

(c) Surface texture

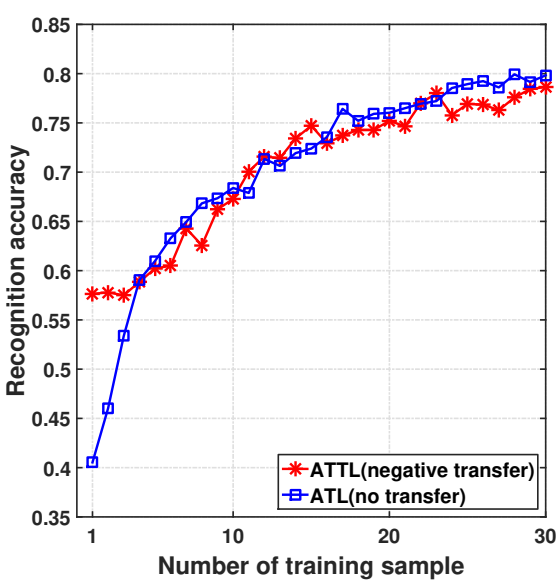

(b) Stiffness

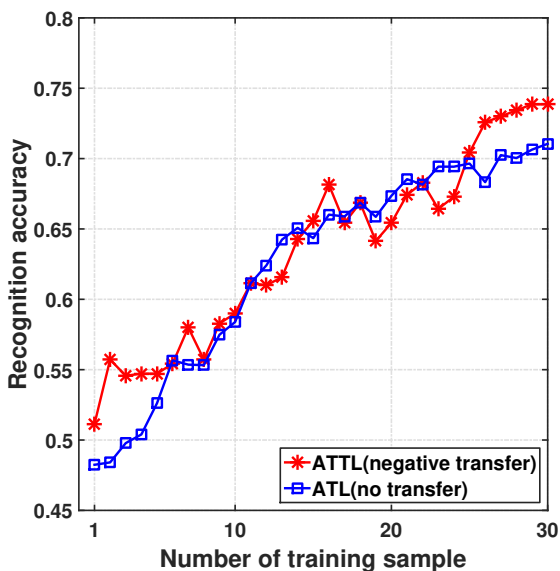

(d) Thermal conductivity

Fig. 15. Evaluation of active tactile learning with negative prior knowledge constructed by deliberately selected five prior objects that were unrelated to the new objects. The blue index indicates the prior objects, and the red index new objects. (a) Learning about the new objects based on three physical properties, prior objects: object $\{2,3,6,10,13\}$; (b) based on only stiffness, prior objects: object $\{1,2,3,10,18\} ;$ (c) based on only surface texture, prior objects: object $\{6,7,9,10,21\}$; (d) based on only thermal conductivity, prior objects: object $\{4,6,8$, $10,13\}$.

strategy to sample the training dataset was needed. Contrary to previous works, using the ATL method the robot strategically selected the next object and next physical property to learn. Therefore, It constructed reliable observation models by applying a fewer exploratory actions on the objects than baselines methods during realtime tactile exploration. In other words, the robot did not deposit any redundant 
tactile information.

By taking benefit of our proposed active tactile transfer learning algorithm (ATTL), the robot was able to re-use its tactile knowledge constructed in advance to learn about new unknown objects. The robot automatically leveraged the most relevant and informative prior knowledge to learn about new unknown objects with a little number of samples or even one with very high recognition accuracy. It achieved $68 \%$ recognition accuracy with only one training sample plus 10 prior tactile knowledge (one-shot tactile learning). Besides, the robot was able to automatically decide how much to leverage the prior tactile knowledge, or stop transferring the irrelevant one which could degrade the learning performance (Fig. 15). Furthermore, the robot attained higher recognition rates, when the number of its prior tactile knowledge increased (Fig. 9 and Fig. 10). This accounts for the fact that increasing the number of prior knowledge also enhances the probability of finding relevant ones (see Fig. 11). The experimental results show that in all scenarios, ATTL outperformed uniform learning strategy in which training data was collected uniformly and no past tactile experience was leveraged. ATTL also performed better than the ATL method, because following ATL the robot was unable to use any prior knowledge, even though it could strategically collect training samples. On the contrary, ATTL can not only intelligently select the exploratory actions to apply on the objects, but also exploit the prior tactile knowledge.

A limiting assumption of our work is that the positions of the experimental objects are fixed, after we have randomly placed them in the unknown workspace. Moreover, due to the low spacial resolution provided by the proximity sensors on the artificial skin array, objects that are close to each other can hardly be clustered after the robot explores the workspace. In order to tackle with this problem, the spacial resolution of the sensor array can be increased by fusing the proximity information and force signals while the robot is contacting with the objects.

In the future, we consider to cover the entire robotic arm with artificial skin to facilitate the robot to explore the workspace in arbitrary directions. Besides, compared to our previous work (Kaboli et al. ${ }^{44,47,48}$ ), our proposed ATTL method enables the robot to actively transfer multiple tactile knowledge (surface texture, stiffness, and thermal conductivity). In both studies, the tactile knowledge is leveraged in the same domain (e.g. transferring the knowledge of stiffness from the prior objects to the new objects). In the future, we will consider to adapt the prior tactile knowledge from different domains (e.g. transferring the prior knowledge of thermal conductivity to help learn about new objects based on stiffness). Furthermore, our experimental results showed that increasing the number of prior knowledge improves the recognition rate and learning performance (Fig. 9 and Fig. 10). Despite this, having too many prior knowledge, e.g. from 1000000 objects, will largely increase the computational complexity so that it takes too long for the robot to select the relevant prior information. Finding a solution to remove such a constrain in any transfer learning approach can be a new interesting challenge to tackle for the future research. Finally, in our future study, we will try to extend our proposed 
framework to transfer tactile knowledge from one robotic platform (e.g. a robotic arm) to another robotic platform (e.g. humanoid robot) that can be equipped with different sensing modalities.

\section{Conclusion}

In this study, we proposed a probabilistic tactile-based framework about active tactile transfer learning for object discrimination in the unknown environment. Using our proposed framework, the robot performed a complete series of tasks to (1) efficiently explore the unknown workspace and determine the number and positions of the objects; (2) actively learn about objects based on their physical properties (stiffness, surface texture, and thermal conductivity); (3) leverage the robot's prior tactile experience and efficiently learn about new objects with a small number of training samples. The effectiveness of our proposed framework was evaluated through online experiments and evaluations. Results shows that the framework outperforms the baseline methods in all tasks. Using the active pre-touch strategy, the robot obtained a maximum workspace uncertainty reduction of $30 \%$ and $70 \%$ compared to uniform and random strategies respectively, and had a better estimation of the object positions. Employing the ATL method, the robot applied 50\% fewer exploratory actions than the baseline strategies to learn about objects and reached the same recognition accuracy. Taking advantage of the attained prior tactile knowledge, the autonomous robot that used the ATTL method could efficiently discriminate among new objects with $10 \%$ and $15 \%$ higher recognition accuracy compared to the ATL and uniform methods.

\section{Video}

The video to this paper can be found in the following link: http://web.ics.ei.tum.de/ mohsen/videos/IJHR2017.mp4

\section{Acknowledgement}

We would like to thank Kunpeng Yao and Zhiliang Wu for their help during experiments.

\section{References}

1. G. Robles-De-La-Torre, The importance of the sense of touch in virtual and real environments, IEEE MultiMedia, pp.24-30 (2006).

2. H. Liu, J. Greco, X. Song, J. Bimbo, and K. Althoefer, Tactile Image Based Contact Shape Recognition Using Neural Network, IEEE I nternational Conference on Intelligent Robots and Systems, pp.2539-2544 (2013).

3. H. Liu, Song, T. Nanayakkara, L. D. Seneviratne, and K. Althoefer, A Computationally Fast Algorithm for Local Contact Shape and Pose Classification using a Tactile Array Sensor, IEEE International Conference on Robotics and Automation, pp.1410-1415 (2012). 
4. Y. Jia, and J. Tian, Surface Patch Reconstruction From 'One-Dimensional' Tactile Data, IEEE Transactions on Automation Science and Engineering, pp.400-407 (2010).

5. M. V. Liarokapis, B. Calli, A. J. Spiers, and A. M. Dollar, Unplanned, model-free, single grasp object classification with underactuated hands and force sensors, IEEE International Conference on Intelligent Robots and Systems, pp.5073-5080 (2015).

6. T. Matsubara and K. Shibata, Active tactile exploration with uncertainty and travel cost for fast shape estimation of unknown objects, Robotics and Autonomous Systems, pp.314-326 (2017).

7. H. Liu, K. C. Nguyen, V. Perdereau, J. Bimbo, J. Back, M. Godden, L. D. Seneviratne, and K. Althoefer, Finger contact sensing and the application in dexterous hand manipulation, Autonomous Robots, pp.25-41 (2015).

8. Nawid Jamali, Carlo Ciliberto, Lorenzo Rosasco, and Lorenzo Natale, Active Perception: Building Objects Models using Tactile Exploration, IEEE International Conference on Humanoid Robots, (2016).

9. P. Dallaire, P. Giguere, D. Edmond, and B. Chaib-draa, Autonomous tactile perception: A combined improved sensing and Bayesian nonparametric approach, Robotics and Autonomous Systems, pp.422-435 (2014).

10. P. Giguere, and G. Dudek, A Simple Tactile Probe for Surface Identification by Mobile Robots, IEEE Transactions on Robotics, pp.534-544 (2011).

11. K. Watanabe, M. Sohgawa, T. Kanashima, M. Okuyama, and H. Norna, Identification of various kinds of papers using multi-axial tactile sensor with micro-cantilevers, World Haptics Conference, pp.139-144 (2013).

12. D. S. Chathuranga, Z. Wang, V. A. Ho, A. Mitani, and S. Hirai, A biomimetic soft fingertip applicable to haptic feedback systems for texture identification, IEEE International Symposium on Haptic Audio Visual Environments and Games, pp.29-33 (2013)

13. N. Jamali, and C. Sammut, Majority Voting: Material Classification by Tactile Sensing Using Surface Texture, IEEE Transactions on Robotics, pp.508-521 (2011).

14. V. Chu, I. McMahon, L. Riano, C. G. McDonald, and Q. He, and others, Using robotic exploratory procedures to learn the meaning of haptic adjectives, IEEE International Conference on Robotics and Automation, pp.3048-3055 (2013).

15. S. J. Lederman, The perception of surface roughness by active and passive touch, Bulletin of the Psychonomic Society, pp.253-255 (1981).

16. T. Bhattacharjee, J. Wade, and C. C. Kemp, Active tactile exploration with uncertainty and travel cost for fast shape estimation of unknown objects, Proceedings of Robotics: Science and Systems, pp.297-304 (2015).

17. Jivko Sinapov, Vladimir Sukhoy, Ritika Sahai, and Alexander Stoytchev, Vibrotactile recognition and categorization of surfaces by a humanoid robot, IEEE Transactions on Robotics, pp.488-497 (2011).

18. H. Hu, Y. Han, A. Song, S. Chen, C. Wang, and Z. Wang, A Finger-Shaped Tactile Sensor for Fabric Surfaces Evaluation by 2-Dimensional Active Sliding Touch, Sensors, pp.4899-4913 (2014).

19. W. W. Mayol-Cuevas, J. Juarez-Guerrero, and S. Munoz-Gutierrez, A first approach to tactile texture recognition, IEEE International Conference on Systems, Man, and Cybernetics, pp.4246-4250 (1998).

20. N. H. H. Mohamad Hanif, P. H. Chappell, A. Cranny, and N. M. White, Surface texture detection with artificial fingers, 37th Annual International Conference of the IEEE Engineering in Medicine and Biology Society, pp.8018-8021 (2015).

21. A. Song, Y. Han, H. Hu, and J. Li, A novel texture sensor for fabric texture measurement and classification, IEEE Transactions on Instrumentation and Measurement, 
pp.1739-1747 (2014).

22. K. E. Friedl, A. R. Voelker, A. Peer, and C. Eliasmith, Human-Inspired Neurorobotic System for Classifying Surface Textures by Touch, IEEE Robotics and Automation Letters, pp.516-523 (2016).

23. N. F. Lepora, M. Evans, C. W. Fox, M. E. Diamond, K. Gurney, and T. J. Prescott, Naive Bayes texture classification applied to whisker data from a moving robot, The International Joint Conference on Neural Networks, pp.1-8 (2010).

24. H. P. Saal, J. A. Ting, and S. Vijayakumar, Active Sequential Learning with Tactile Feedback, AISTATS, pp.677-684 (2010).

25. Danfei Xu, Gerald E. Loeb, and Jeremy A. Fishel, Tactile identification of objects using Bayesian exploration, IEEE International Conference on Robotics and Automation, pp.3056-3061 (2013).

26. Nathan F. Lepora, Uriel Martinez-Hernandez, and Tony J. Prescott, Active touch for robust perception under position uncertainty, IEEE International Conference on Robotics and Automation, pp.3020-3025 (2013).

27. Alexander Schneider, Jürgen Sturm, Cyrill Stachniss, Marco Reisert, Hans Burkhardt, and Wolfram Burgard, Object identification with tactile sensors using bag-of-features, IEEE International Conference on Intelligent Robots and Systems, pp.243-248 (2009).

28. R. Martins, J. F. Ferreira, and J. Dias, Touch attention Bayesian models for robotic active haptic exploration of heterogeneous surfaces, International Conference on Intelligent Robots and Systems, pp.1208-1215 (2014).

29. Daisuke Tanaka, Takamitsu Matsubara, Kentaro Ichien, and Kenji Sugimoto, Object manifold learning with action features for active tactile object recognition, IEEE International Conference on Intelligent Robots and Systems, pp.608-614 (2014).

30. S. J. Pan and Q. Yang, A Survey on Transfer Learning, IEEE Transactions on Knowledge and Data Engineering, pp.1345-1359 (2010).

31. C. Goring, E. Rodner, A. Freytag, and J. Denzler, Nonparametric Part Transfer for Fine-grained Recognition, The IEEE Conference on Computer Vision and Pattern Recognition (CVPR), pp. (2014).

32. G. Skolidis and G. Sanguinetti, Semisupervised Multitask Learning With Gaussian Processes, IEEE Transactions on Neural Networks and Learning Systems, pp.12101$2112(2013)$.

33. L. Fei-Fei, R. Fergus, and P. Perona, One-Shot Learning of Object Categories, IEEE Transactions on Pattern Analysis and Machine Intelligence, pp. 594-611 (2006).

34. S. I. Lee, V. Chatalbashev, D. Vickrey, and D. Koller, Learning a meta-level prior for feature relevance from multiple related tasks, In Proceedings of the 2007 international conference on Machine learning (ICML07), pp. 489-496 (2007).

35. A. Vedaldi, G. Guidi, and S. Soatto, Joint Alignment up to (Lossy) Transformations, In Proceedings of the 2008 IEEE Conference on Computer Vision and Pattern Recognition (CVPR08), pp. (2008).

36. V. Jain and E. Learned-Miller, Online Domain Adaptation of a Pre-Trained Cascade of Classifiers, In Proceedings of the 2008 IEEE Conference on Computer Vision and Pattern Recognition (CVPR11), pp. (2011).

37. R. Salakhutdinov, A. Torralba, and J. Tenenbaum, Learning to Share Visual Appearance for Multiclass Object Detection, In Proceedings of the 2008 IEEE Conference on Computer Vision and Pattern Recognition (CVPR11), pp. (2011).

38. R. Urtasun, A. Quattoni, N. D. Lawrence, and T. Darrell, Transfer learning for reinforcement learning domains: A survey, The Journal of Machine Learning Research, pp.1633-1685 (2011).

39. C. Do and A. Ng, Transfer learning for text classification, In Advances in Neural 
Information Processing Systems, pp. 299-306 (2006).

40. Q. Wu, X. Zhou, Y. Yan, H. Wu, and H. Min, Online transfer learning by leveraging multiple source domains. Knowledge and Information Systems, In Knowledge and Information Systems, pp. 1-21 (2017).

41. J. T. Zhou, J .P. Sinno, W. T. Ivor, and H. Shen-Shyang, Transfer Learning for Cross-Language Text Categorization through Active Correspondences Construction, In $A A A I$, pp. 2400-2406 (2016).

42. V. Jayaram, M. Alamgir, Y. Altun, B. Scholkopf, and M. Grosse-Wentrup, Transfer learning in brain-computer interfaces, Pattern Recognition, pp. 20-31 (2016).

43. J. W. Tao, D. Song, S. Wen, Wenjun Hu, Transfer Learning for Deep Learning on Graph-Structured Data, Pattern Recognition, pp. 47-65 (2017).

44. M. Kaboli, T. De La Rosa, R. Walker, and G. Cheng, Re-using prior tactile experience by robotic hands to discriminate in-hand objects via texture properties, IEEE International Conference on Robotics and Automation (ICRA), pp.2242-2247 (2016).

45. P. Mittendorfer and G. Cheng, Humanoid Multimodal Tactile-Sensing Modules, IEEE Transactions on Robotics, pp.401-410 (2011).

46. M. Kaboli, R. Walker, and G. Cheng, In-hand object recognition via texture properties with robotic hands, artificial skin, and novel tactile descriptors, IEEE International Conference on Humanoid Robots, pp.2242-2247 (2015).

47. Mohsen Kaboli, Philipp Mittendorfer, Vincent Hügel, and Gordon Cheng. Humanoids learn object properties from robust tactile feature descriptors via multi-modal artificial skin. IEEE International Conference on Humanoid Robots (Humanoids), pages 187$192,2014$.

48. Mohsen Kaboli and Gordon Cheng. Robust tactile descriptors for discriminating objects from textural properties via artificial robotic skin. IEEE Transactions on Robotics Journal, under review.

49. P. Lanillos, E. Besada-Portas, J. Lopez-Orozco, A. de la Cruz, and M. Jesus, Minimum time search in uncertain dynamic domains with complex sensorial platformsActive Sequential Learning with Tactile Feedback, Sensors, pp.14131-14179 (2014).

50. C. E. Rasmussen, and C. K. I. Williams, Gaussian Processes for Machine Learning, the MIT Press, (2006).

51. Chai, and Kian M, Generalization errors and learning curves for regression with multitask Gaussian processes, Advances in neural information processing systems, pp.279287 (2009).

52. Mohsen Kaboli, Kunpeng Yao, Gordon Cheng. Tactile-based manipulation of deformable objects with dynamic center of mass. IEEE International Conference on $\mathrm{Hu}$ manoid Robots (Humanoids), pages 752-757, 2016.

53. M. Kaboli, D. Feng, K. Yao, P. Lanillos, and G. Cheng. A tactile-based framework for active object learning and discrimination using multimodal robotic skin. IEEE Robotics and Automation Letters, 2(4):2143-2150, 2017.

54. Kunpeng Yao, Mohesn Kaboli, and Gordon Cheng. Tactile-based object center of mass exploration and discrimination. IEEE International Conference on Humanoids Robot (Humanoids), authors with the same contribution, 2017.

55. M. Kaboli and G. Cheng. Novel tactile descriptors and a tactile transfer learning technique for active in-hand object recognition via texture properties. IEEE International Conference on Humanoid Robots (Humanoids)-workshop on tactile sensing for manipulation: new progress and challenges, 2016

56. Mohesn Kaboli. Leveraging over prior knowledge for online learning of visual categories across robots. Thesis, KTH, The Royal Institute of Technology, Stockholm Sweden, 2012 . 
57. Mohsen Kaboli, Alex Long, and Gordon Cheng. Humanoids learn touch modalities identification via multi-modal robotic skin and robust tactile descriptors. Advanced Robotics, 29(21):1411-1425, 2015.

58. T. Tommasi, F. Orabona, M. Kaboli, and B. Caputo. Leveraging over prior knowledge for online learning of visual categories. British Machine Vision Conference (BMVC), 2012.

59. N. Yogeswaran, W. Dang, W.T. Navaraj, and et. al. New materials and advances in making electronic skin for interactive robots. Advanced Robotics, 29(21):1359-1373, 2015.

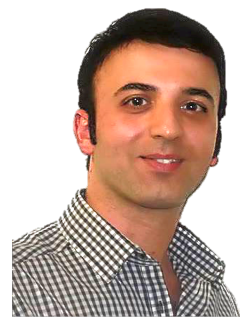

Mohsen Kaboli received his bachelor degree in electrical and electronic engineering and master degree in signal processing and machine learning from the Royal Institute of Technology (KTH) in Sweden, in 2011. In March 2012, he received a scholarship from the Swiss National Foundation for 18 months in order to continue his research as a research assistant at the Idiap lab, Ecole Polytechnique Federale de Lausanne (EPFL).

April 2013, he was awarded a three-year Marie Curie scholarship. Since that time he has been at the Institute for Cognitive Systems directed by Prof. Gordon Cheng in Technical University of Munich (TUM). In November 2013, he visited the Shadow Robot Company for two months. Mohsen has been a visiting researcher at the Human Robotics lab, department of Bioengineering at Imperial College London supervised by Prof. Ettien Burdet from February till April 2014. From September 2015 till January 2016, he spent 5 months as a visiting research scholar at the Intelligent Systems and Informatics lab at the University of Tokyo, Japan under the supervision of Prof. Yasuo Kuniyoshi.

Di Feng was born in Kunming, China, in 1991. He is currently pursuing the masters degree in electrical and computer

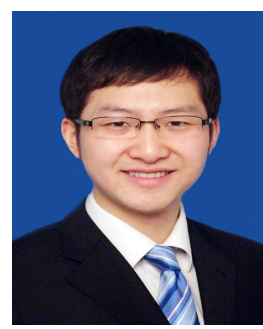
engineering at the Technical University of Munich. He received the bachelors degree in mechatronics with honor from Tongji University, in 2014. During his bachelor study, he received DAAD scholarship and visited Niederrhein University of Applied Science as exchange student from Sep. 2013 till Aug 2014. His current research is centered on robotic active learning and exploration through tactile sensing. He is interested in perception, lifelong learning machines and cognitive systems. 


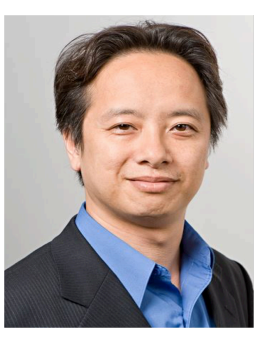

Gordon Cheng is the Professor and Chair of Cognitive Systems, and founding Director of the Institute for Cognitive Systems, Technical University of Munich, Germany. He was the Head of the Department of Humanoid Robotics and Computational Neuroscience, ATR Computational Neuroscience Laboratories, Kyoto, Japan, from 2002 to 2008. He was the Group Leader for the JST International Cooperative Research Project, Computational Brain, from 2004 to 2008. He was designated a Project Leader from 2007 to 2008 for the National Institute of Information and Communications Technology of Japan. He has held visiting professorships worldwide in multidisciplinary fields comprising mechatronics in France, neuroengineering in Brazil, and computer science in the USA. He held fellowships from the Center of Excellence and the Science and Technology Agency of Japan. Both of these fellowships were taken at the Humanoid Interaction Laboratory, Intelligent Systems Division at the Electrotechnical Laboratory, Japan. He received the Ph.D. degree in systems engineering from the Department of Systems Engineering, The Australian National University, in 2001, and the bachelor degrees in computer science from the University of Wollongong, Wollongong, Australia, in 1991 and 1993, respectively. He was the Managing Director of the company G.T.I. Computing in Australia. His current research interests include humanoid robotics, cognitive systems, brain machine interfaces, biomimetic of human vision, human-robot interaction, active vision, and mobile robot navigation. $\mathrm{He}$ is the co-inventor of approximately 15 patents and has co-authored approximately 180 technical publications, proceedings, editorials, and book chapters. 